\title{
POINT-OF-INTEREST RECOMMENDATION IN LOCATION-BASED SOCIAL NETWORKS
}

\author{
by
}

\author{
Alireza Pourali \\ A thesis \\ presented to Ryerson University \\ in partial fulfillment of the \\ requirements for the degree of \\ Master of Applied Science \\ in the program of
}

Bachelor of Engineering, Ryerson University of Toronto, 2016

Electrical and Computer Engineering

Toronto, Ontario, Canada, 2018

(C) Alireza Pourali, 2018 


\section{AUTHOR'S DECLARATION FOR ELECTRONIC SUBMISSION OF A THESIS}

I hereby declare that I am the sole author of this thesis. This is a true copy of the thesis, including any required final revisions, as accepted by my examiners.

I authorize Ryerson University to lend this thesis to other institutions or individuals for the purpose of scholarly research.

I further authorize Ryerson University to reproduce this thesis by photocopying or by other means, in total or in part, at the request of other institutions or individuals for the purpose of scholarly research.

I understand that my dissertation may be made electronically available to the public. 


\title{
POINT-OF-INTEREST RECOMMENDATION IN LOCATION-BASED SOCIAL NETWORKS
}

\author{
Master of Applied Science, 2018 \\ Alireza Pourali \\ Electrical and Computer Engineering \\ Ryerson University
}

\begin{abstract}
The focus of point-of-interest recommendation techniques is to suggest a venue to a given user that would match the users' interests and is likely to be adopted by the user. Given the multitude of the available venues and the sparsity of user check-ins, the problem of recommending venues has shown to be a difficult task. Existing literature has already explored various types of features such as geographical distribution, social structure and temporal behavioral patterns to make a recommendation. In this thesis, we show how a comprehensive set of user and venue related information can be methodically incorporated into a heterogeneous graph representation based on which the problem of venue recommendation can be efficiently formulated as an instance of the heterogeneous link prediction problem on the graph and we propose a new set of features derived based on the neural embeddings of venues and users. We additionally show how the neural embeddings for users and venues can be jointly learnt based on the prior check-in sequence of users and then be used to define a set of new features. We have also used a new proposed heterogeneous graph similarity search framework to find similarity between users and venues using our graph. These features are integrated into a feature-based matrix factorization model. Our experiments show that the features defined over the user and venue embeddings are effective for venue recommendation and outperform existing state of the art methods.
\end{abstract}




\section{Acknowledgements}

I would like to express my special thanks to my supervisor, Dr.Ebrahim Bagheri of the Department of Electrical, Computer, and Biomedical Engineering at Ryerson University. Dr.Bagheri was always available whenever I needed assistance about my research and projects. The completion of my adventure at Ryerson University could not have been possible without Dr. Bagheri's guidance and helpful suggestions.

I would also like to express my deep appreciation to Dr. Fattane Zarrinkalam, another contributor to my thesis work who also helped me in completing this project and all of my lab mates from Laboratory for Systems, Software and Semantics at Ryerson University. Finally, I would like to thank my family Mr. and Mrs. Pourali, Nazanin Pourali, Mohammad Ali Tabatabaee and Mahtab Tamannaee, for providing support throughout my journey at Ryerson. This couldn't have been possible without you all and I dedicate this thesis to you.

Alireza Pourali 


\section{Contents}

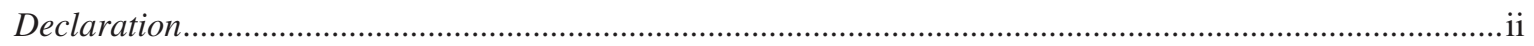

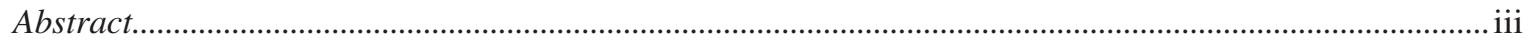

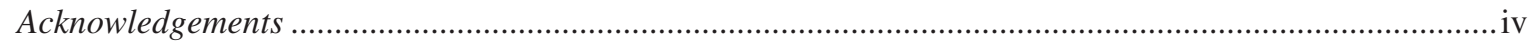

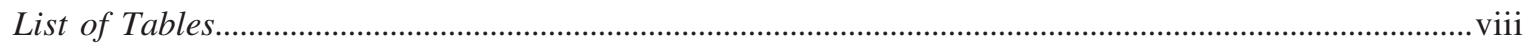

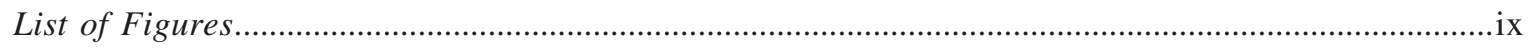

1 Introduction $\quad 1$

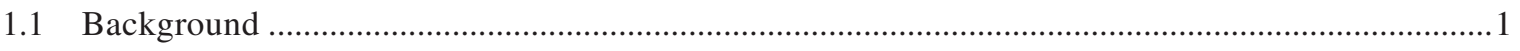

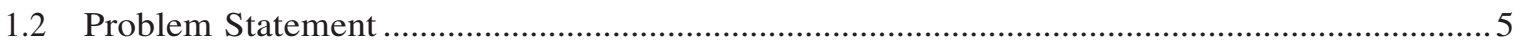

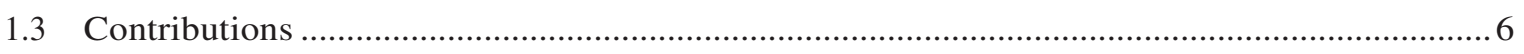

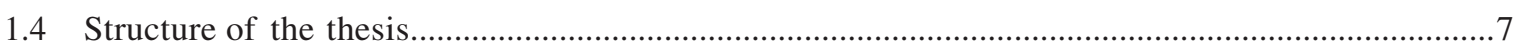

2 Literature Review $\quad 8$

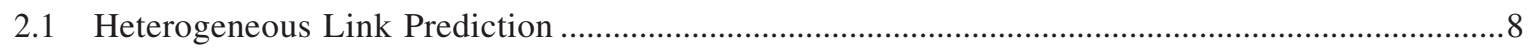

2.1.1 Heterogeneous Information Networks ………..........................................................

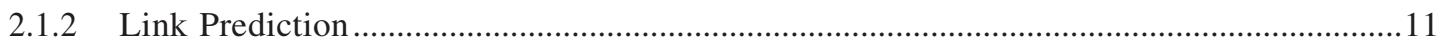

2.1.3 Topological Features in Heterogeneous Information Networks ..........................................12

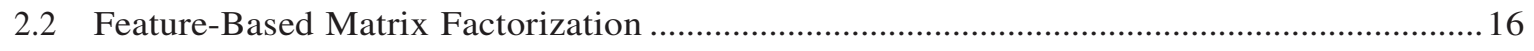

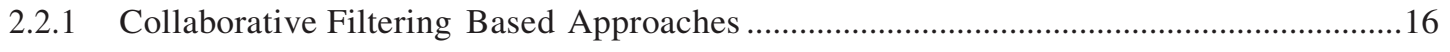




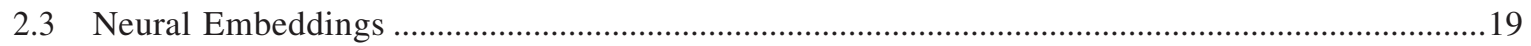

2.4 Point-of-Interest Recommendation .......................................................................... 20

2.4.1 Pure Check-in Data Based Point-of-Interest Recommendation ...............................20

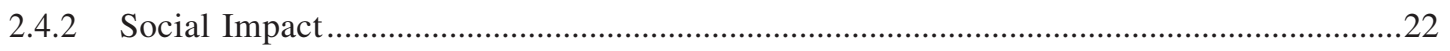

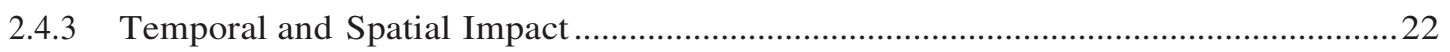

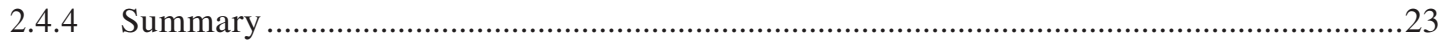

3 The Proposed Approach 24

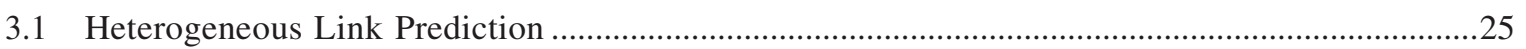

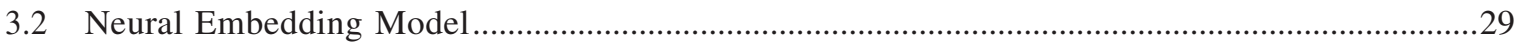

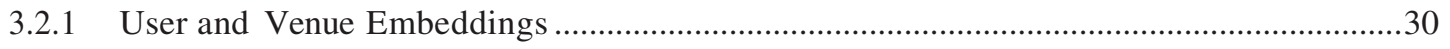

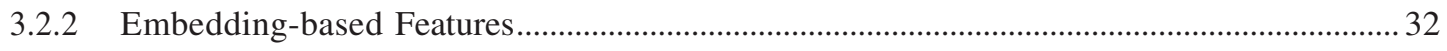

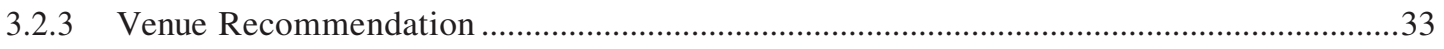

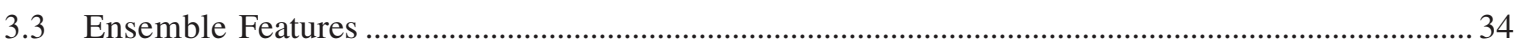

4 Empirical Evaluations 38

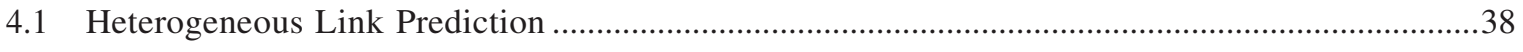

4.1.1 Dataset and Experimental Setup .............................................................. 38

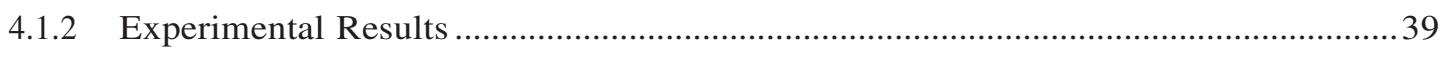

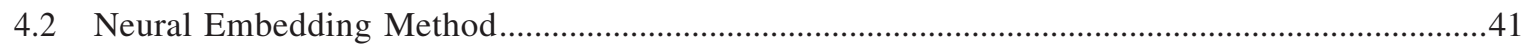

4.2.1 Dataset and Evaluation Methodology ...............................................................41

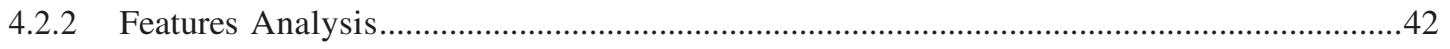

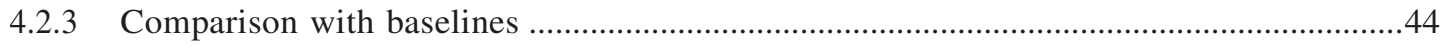

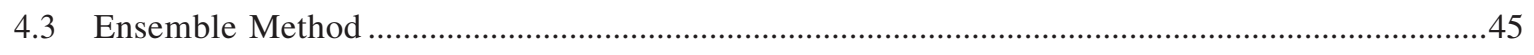

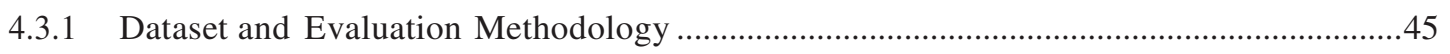

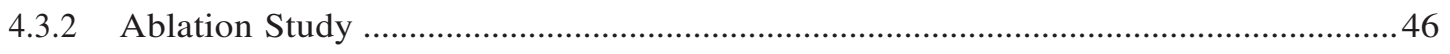

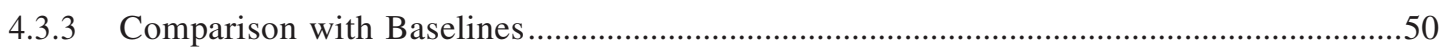


5 Conclusion

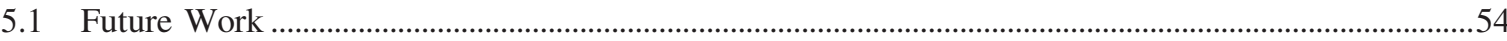

References 


\section{List of Tables}

2.1 Heterogeneous Information Network Meta Paths and Their Meanings

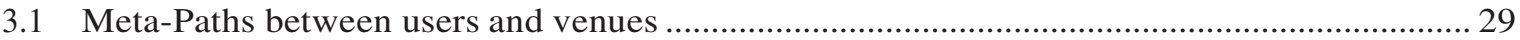

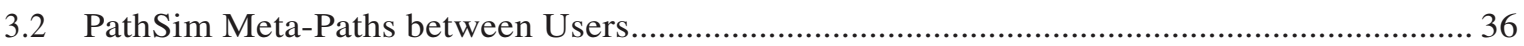

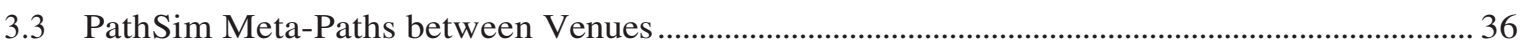

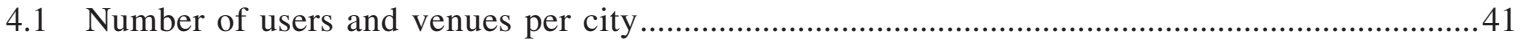

4.2 Five variants based on our proposed features ............................................................................46

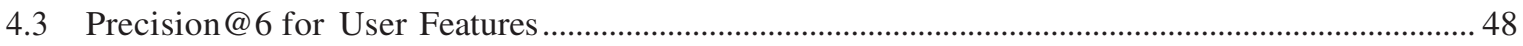

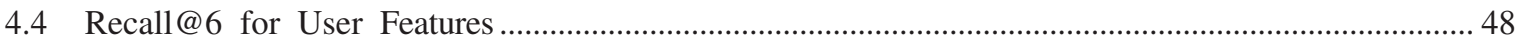

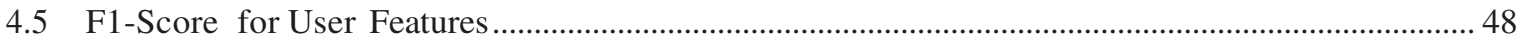

4.6 Precision @ 6 for Venue Features ................................................................................................................. 49

4.7 Recall@6 for Venue Features........................................................................................................................ 49

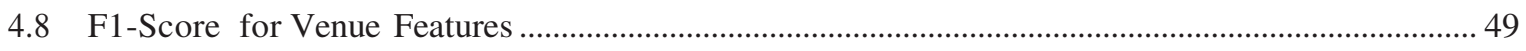

4.9 Precision@6 for User-Venue Similarity Features...................................................................................50

4.10 Recall @ 6 for User-Venue Similarity Features.......................................................................................... 50

4.11 F1-Score for User-Venue Similarity Features.................................................................................... 50

4.12 All models comparative analysis with the baselines divided across different cities................. 51 


\section{List of Figures}

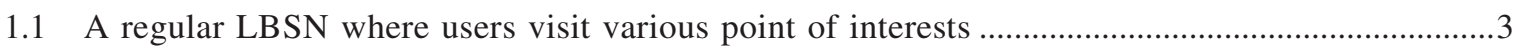

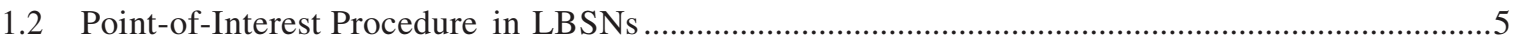

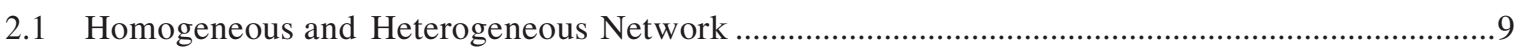

2.2 Heterogeneous Information Network for DBLP Bibliographic Network ……………………......12

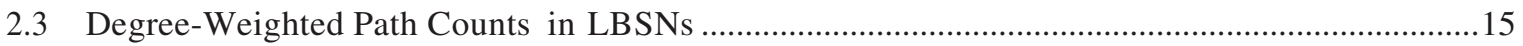

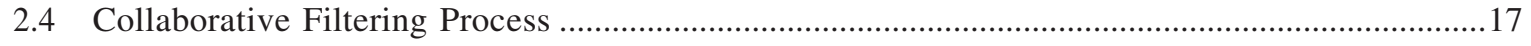

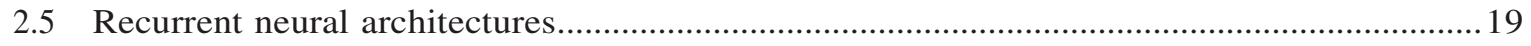

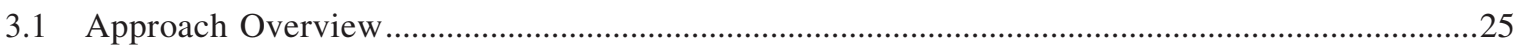

3.2 Sample heterogeneous graph representation of LBSN data.........................................................26

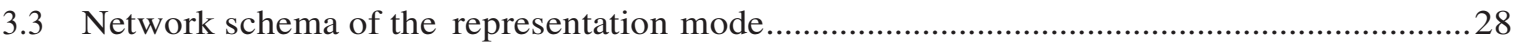

3.4 A framework for learning user and venue embeddings in the same feature space ......................30

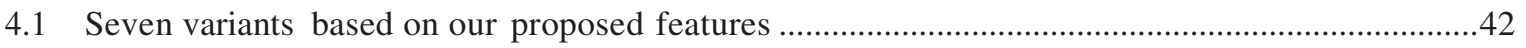

4.2 The Precision@ 6 of the seven variants of our proposed approach..................................................43

4.3 The Recall@ 6 of the seven variants of our proposed approach........................................................43 


\section{Chapter 1}

\section{Introduction}

\section{$1.1 \quad$ Background}

With a significant number of users, Location-Based Social Networks (LBSN) such as Facebook Places, Yelp, and Foursquare are starting to be well known as a result of their capability to share location related information. Until August 2018, Foursquare users have generated over 12 billion check-ins over more than 105 million spots mapping organizations across the world. Also, at least fifty million users use Foursquare City Guide along with Foursquare Swarm every month. ${ }^{1}$ LBSNs collect users' check-in info such as visited locations' geographical information (longitude and latitude) and also users' comments about a location.

Obtaining information on human spatiotemporal actions is a perennial study. Today, as a result of its abundant potential applications, this particular information is becoming increasingly more important. The recent years have experienced a significantly improved way of life of modern society. Together with the ubiquitous access to the web and the popularity of different kinds of location-based social networking sites (LBSN) including the microblogging service Twitter or even the check-in service Foursquare, most

\footnotetext{
${ }^{1}$ (https://foursquare.com/about)
} 
people are progressively prepared to report the personal experiences of theirs on the LBSNs from their immediate vicinity in all sorts of scenarios.

In these services, users could easily share their geospatial Point of Interests and its related contents in the actual physical community by using web-based platforms. For example, an individual with a cell phone is able to share feedback with the friends regarding a restaurant at which she's dined. Different users can expand their social networks by using friend recommendations produced from overlapped location records. For instance, individuals who regularly play soccer at the same soccer field could be placed in contact. The location dimension bridges the gap between the actual physical world as well as the digital online social network providers, providing rise to unique challenges and opportunities within traditional recommender models within the following aspects:

(i) Abundant knowledge: A point of interest is among the most crucial elements determining a user's context. Extensive information about a user's preferences and behavior may be learned by using their location history [49]. The enormous amount of location related statistics generated by consumers enhances the chance that social opinions can be can be effectively evaluated by recommender techniques.

(ii) Complex relations and objects: A location is a unique object in location based social networks (LBSNs), producing unique associations between individuals, point of interests, and also locations and users. New recommendation scenarios, like location and itinerary suggestions, can certainly be allowed by using this type of information, while with traditional recommendation scenarios, like friend and advertising recommendation, can be improved. Nevertheless, these recommendations demand new techniques for producing high-quality suggestions.

In an effort to enhance user experience in LBSNs, Point-of-Interest (POI) recommendation can be proposed which suggests locations for people to check out from mining users' social relationships and check-in records. The following figure shows an ordinary LBSN, exhibiting the interactions (e.g., check- 
in activity) between POIs (also called venues) and users. There are two types of nodes, locations, and users. An edge starting right from a person and ending at point of interest can indicate the user's traveling histories, and the weight of the edge is able to indicate the number of visits or perhaps the user's feedback ratings.

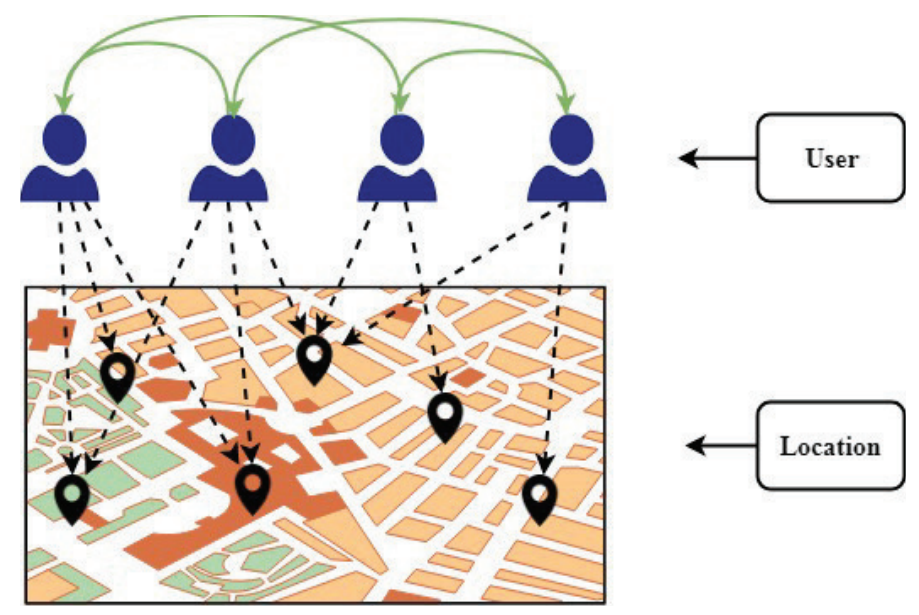

Figure 1.1: A regular LBSN where users visit various point of interests.

POI recommendation is among the more important tasks in LBSNs that enables users to explore new places inside the LBSNs. POI recommendation commonly mines users' check-in records, venue info including categories, plus users' interpersonal interactions to suggest a list of POIs where users will probably check-in later on. POI recommendation does not just improve the user viscosity to LBSN service distributors, but additionally benefits advertising organizations with an efficient method of launching ads to potential customers. Notably, users are able to explore restaurants that are nearby and shopping malls in Foursquare. However, by using effective POI recommendation models, merchants are in a position to help users quickly find them.

Point-of-interest (POI) recommendation has been investigated recently by researchers and it is among the more interesting tasks that enhance user participation on the social network. In the past few years, researchers have designed and discovered various POI recommendation models to increase the accuracy 
of the recommended venues to the users. The simple idea behind this is to recommend a set of locations to the user based on her historical visits. These visits are also called check-ins in the social networks where the user will generate footprints of the locations she has visited and/or left a comment or a review for that location. This task has been well studied using Location-based Social Networks (LBSNs) which are social networks that use smart devices GPS for broadcasting the users' location and other content from their mobile device. Due to the growing popularity of LBSNs, a high volume of user location information has been generated [46].

Due to the growing popularity of LBSNs, a high volume of user location information has been generated [46] and as such, point-of-interest (also called venue) recommendation models have received attention by researchers in the past few years and many providers are now focused on developing such recommendation systems for their end-users to facilitate social interaction [26]. Due to this, researchers have focused on analyzing these generated social data that are related to users point-of-interests (POI) based on their preferences and personalities.

In this thesis, we take a different perspective on the problem of Point-of-Interest recommendation by formalising user LBSN info in the style of a heterogeneous graph and proposing a new set of features based neural embeddings of users and venues and the metrics generated from the graph [33]. We propose how the problem of point-of-interest recommendation can be considered as an instance of the link prediction problem on heterogeneous graphs. In our proposed approach, these newly derived features are integrated into a feature-based matrix factorization model for Point-of-Interest recommendations. Our experiments demonstrate that the features outlined across the user and venue embeddings are effective for venue recommendation and outperform current state of the art approaches. 


\subsection{Problem Statement}

In this thesis, we take a different perspective on the problem of Point-of-Interest recommendation by formalizing user LBSN information in the form of a heterogeneous graph, and we propose a new set of features based on the neural embedding of users and venues. A set of features are integrated into a feature-based matrix factorization model for more accurate recommendations. We have proposed the following models for the problem of POI recommendation and investigated and compared their performance:

1. Heterogeneous Link Prediction Model

2. Neural Embedding Model

3. Ensemble Model

POI recommendation seeks to mine users' check-in data and recommend POIs for the individuals in LBSNs. POI recommendation aims to recommend a user a listing of POIs through available check-in data.

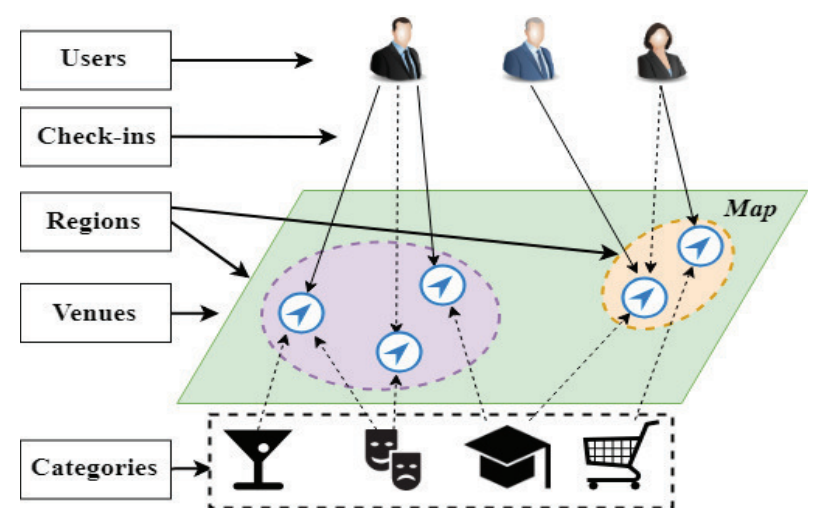

Figure 1.2: Point-of-Interest Procedure in LBSNs. 
There is a high amount of useful data in user check-ins which can be used for Point-of-Interest recommendation. As illustrated in Fig 1.2, users, venues, regions and categories of venues are rich information for making more accurate recommendations. Also, as it can be seen, black bolded lines indicate users historical check-ins and dashed lines are for recommendations made to the user. For instance, user "Alex" is a friend of "Joe" and "Alex" plays soccer at the "Trio Sportsplex" venue many times. If "Joe" is also interested in playing Soccer, "Trio Sportsplex" will be recommended to him and there is a high chance that "Joe" may visit that venue in the future.

To describe the problem more formally, assume a check-in (digital footprint that users leave after visiting a location) is denoted as a triple $(u, v, t)$ that indicates a user $u$ heading to POI $v$ at time $t$. Given the check-in sequences, POI recommendation seeks to recommend a POI list for every user $u$. A variety of locations are recommended to the user and in case she is keen on one of them and visits that place, the most effective results are attained. The dataset for this certain problem consists of users and location identification, categories of the locations and the time of the visits.

\subsection{Contributions}

In this thesis, we provide the following major contributions:

1. We show how a collection of LBSN information including user relationships, past user-venue interaction history, venue category information and geographical coordinates can be unified into and represented as a heterogeneous graph. How meta-paths can be extracted from such a heterogeneous graph representation in order to identify potential links between users and venues (points-of-interest).

2. We systematically show how the check-in sequence of users can be utilized to learn neural embeddings for both users and venues within the same embedding space. We introduce three classes of features based on neural embeddings of users and places, which capture the characteristics of 
users, locations, and their interactions.

3. We also show how the embedding-based and metapath-based similarity is used to find the similarity between users and venues by utilizing the generated embeddings and defined meta-paths, which lead to higher accuracy recommendations to the users. We formalize how the defined features are encoded into feature-based matrix factorization for venue recommendation and extensively compare our work with the state-of-art.

\subsection{Structure of the thesis}

This thesis is prepared as follow:

1. Chapter 2 - Literature Review: This chapter covers the details of heterogeneous link prediction, feature-based matrix factorization and Point-of-Interest recommendation techniques.

2. Chapter 3 - The Proposed Approach: This chapter describes the proposed approaches for the problem of Point-of-Interest recommendation using three different techniques of Heterogeneous Link Prediction, Feature-based Matrix Factorization and Ensemble of these methods through an ablation study.

3. Chapter 4 - Empirical Evaluations: This chapter focuses on the through evaluations performed on the three proposed approaches and also the weaknesses along with strengths of them are reviewed.

4. Chapter 5 - Conclusion: In this chapter we summarize the proposed strategies and talk about potential future work. 


\section{Chapter 2}

\section{Literature Review}

In this chapter, we present an introduction of the models used in our work including Heterogeneous Link Prediction, Feature-based matrix factorization followed by the state-of-the-art in Point-of-Interest recommendation.

\subsection{Heterogeneous Link Prediction}

\subsubsection{Heterogeneous Information Networks}

Information Networks: An information network is described as a directed graph $G=(V, E)$ where each object $v \in V$ belongs to one specific object form in the object type set, and each link $e \in E$ belongs to one specific relation type in the relation type set.

Heterogeneous Information Networks: An information network is called heterogeneous information network in case the types of objects or the types of relations are larger than one; if not, it is known as a homogeneous information network. The majority of contemporary information network analysis techniques use a simple assumption: the types of objects and links are unique [38] where the networks are generally homogeneous filled with identical type of links and objects as investigated in most link 
prediction studies [12], [20], [41]. However, since we have multiple objects in our work (users, venues, categories, etc.) which will be covered in the following chapter, homogeneous information network cannot be a helpful solution. As compared to widely used homogeneous information networks, heterogeneous information networks can easily fuse a lot more information and contain richer semantics within links and nodes, as seen in Figure 2.1.

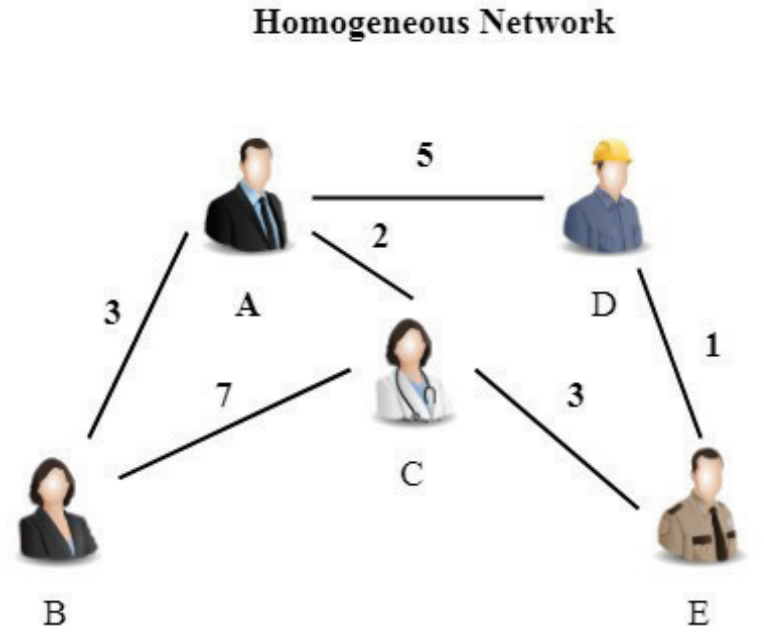

Authors Network

\section{Heterogeneous Network}

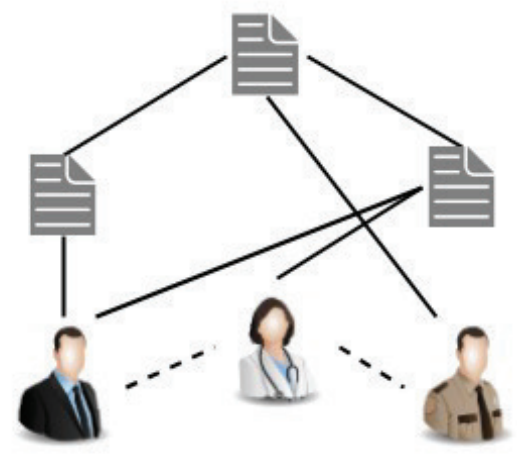

Author-Paper Network

Figure 2.1: Homogeneous and Heterogeneous Network

Since the introduction of the idea of heterogeneous information network in [37], more and more researchers have recognized the significance of Heterogeneous Information Network Analysis (HINA) and many studies have been based on this idea $[40,13,33,39]$. In addition, since most of the networks in the real word contain multiple types of objects and links, homogeneous networks does not give us the ability to construct effective recommendation systems due to the multiple types of objects available in the digital footprints. For example, a shopping network such as Amazon, contains information about the types of products, companies, users, and reviews, with links from types of buy/bought, sell/sold, 
review/reviewed and so on.

Metapath Methodology: Distinct from homogeneous networks, in heterogeneous networks objects can be connected via various paths and each path has a different meaning. For example, Table 2.1 shows various meta-path instances with their meanings. These paths could in fact be classified as meta paths [37] where a metapath $P$ is a path defined on a Schema $S=(N, E)$. Each metapath starts with a source node and ends with a target node. The length of each metapath is not limited and each one can be up to $n$ hops long. In addition, meta-paths are abbreviated using the first letters of paths. For example, as described in Table 2.1, Metapath Author $\stackrel{\text { write }}{\longrightarrow}$ Paper $\stackrel{\text { published-at }}{\longrightarrow}$ Venue (APV) has a source node of Author and target node of Venue or the authors publishing a paper in the same venue is denoted as AVA. A meta path $\mathrm{P}$ is a symmetric path, when the relation $\mathrm{R}$ outlined by it's symmetric (i.e., $P$ is the same as $P^{-1}$ ), like AVA.

\begin{tabular}{|c|c|c|}
\hline Path Instance & Meta-path & Meaning \\
\hline Jiawei - EDBT - Tianyi & Author - Venue - Author (AVA) & Authors publish a paper in the same venue \\
\hline Jiawei - CIKM - Tianyi & Author - Venue - Author (AVA) & Authors publish a paper in the same venue \\
\hline Jiawei - ROPA - EDBT & Author - Paper - Venue (APV) & Author publish a paper at a venue \\
\hline
\end{tabular}

Table 2.1: Heterogeneous Information Network Meta Paths and Their Meanings.

The heterogeneity of objects along with links makes it difficult to work with known topological principles in homogeneous networks for algorithmic design. For instance, the number of the common neighbors is regularly employed as a feature for link prediction in homogeneous networks. Nevertheless, in heterogeneous networks, the neighbors of an item can come from various types, and the amount of shared neighbors is not in a position to completely represent this heterogeneity. The recent work of [37] has drawn a lot of attention due to its developed methodology of PathPredict. In this paper, Sun et al examined the challenge of co-author relationship prediction in the heterogeneous bibliographic network 
and solved it by using the meta path-based PathPredict relationship prediction model which will be covered in subsection 2.1.3.

\subsubsection{Link Prediction}

Link prediction is an essential dilemma of link mining which attempts to estimate the chance of the presence of a link between two nodes, based on found links and also the features of the nodes. The majority of the current link prediction research studies are intended for homogeneous networks, where only one kind of objects is present in the network [20], [41]. Link prediction is commonly considered a simple binary classification problem: for any kind of 2 possibly linked objects, predict whether or not the link exists (1) or otherwise (0). Link prediction in an Heterogeneous Information Network has continued to be an important research subject in the recent years, that has the following qualities. To begin with, the links being predicted are of different types, because objects in HIN are linked with various links types. Next, there are dependencies existing among several forms of links. Hence link prediction in an Heterogeneous Information Network needs to predict various links types collectively by capturing the complex and diverse relationships among different sorts of links and using the complementary prediction information. For example, Fig 2.2 presents a collective prediction dilemma of numerous links types in Heterogeneous Information Network. As seen, there are four types of nodes in this network (Topic, Paper, Author and Venue) and six types of edges (contain, cite, write, publish and mention) between these nodes.

One sort of approach is making this prediction solely based on structural characteristics of the network as also discussed in Liben-Nowell's [23] survey of predictors on various graph proximity measures. A different type of approach is making use of attribute information for link prediction. For instance, in our work a structured logistic regression model has been trained by using relational features of users digital footprints to predict potential links between users and point-of-interests in future which will be covered 


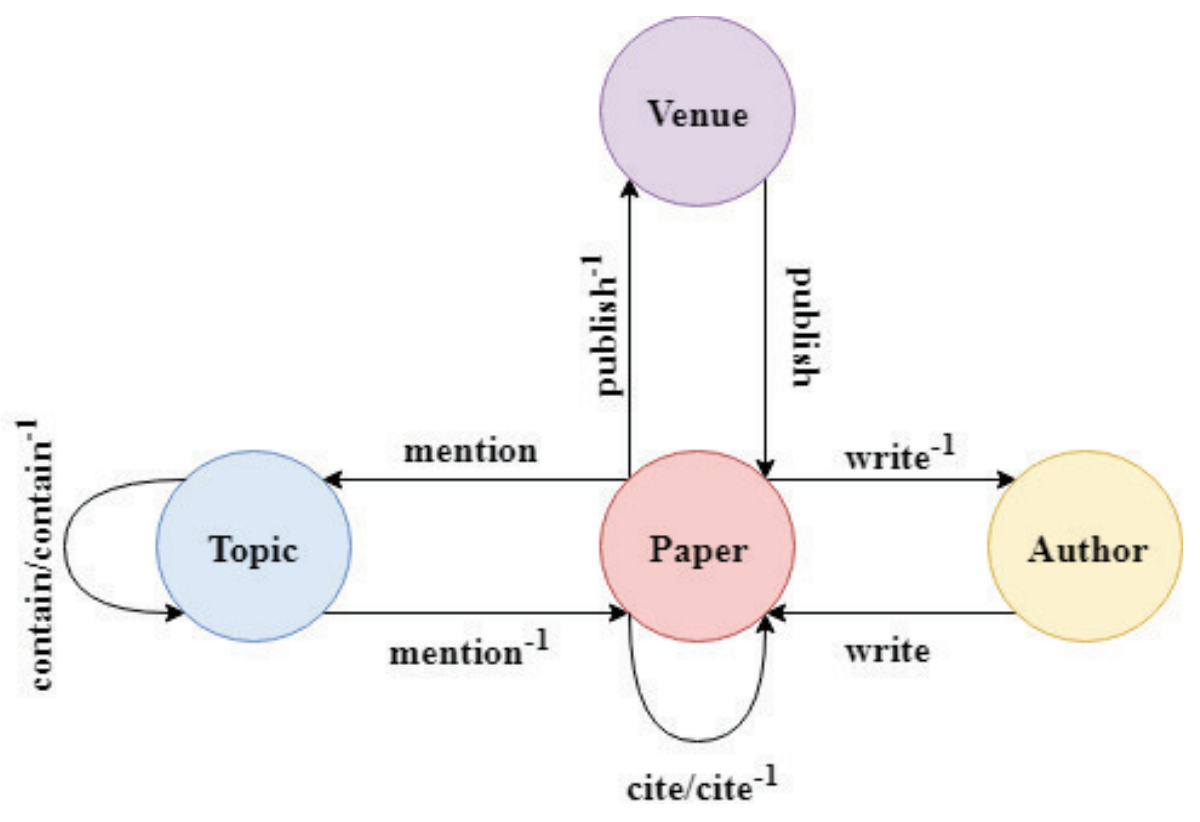

Figure 2.2: Heterogeneous Information Network for DBLP Bibliographic Network

in Chapter 3.

Similar to our work, many researches in this field have employed a three-step process to find solutions to the link prediction problem in Heterogeneous Information Networks.

1. Define a set of meta-paths from the Heterogeneous Information Network

2. Extract meta-path based feature vectors (covered in Chapter 2.2.3)

3. Train a logistic regression model to compute the presence likelihood of a link

\subsubsection{Topological Features in Heterogeneous Information Networks}

Topological features are also called structural features, which aim at extracting connectivity characteristics for pairs of objects. Topological feature based link prediction aims at inferring the upcoming connectivity by using the present connectivity of the network. There are a handful of often used topological features outlined in homogeneous networks, including the number of common neighbors [16], [29]. 
We first review several commonly used topological features in homogeneous networks, and then review the systematic meta path-based methodologies to define topological features in heterogeneous networks.

\section{Homogeneous Information Networks Topological Features:}

1. Jaccard's coefficient: Jaccard's coefficient is a measure to assess the similarity among 2 neighbor sets, which can be considered the normalized selection of common neighbors.

2. Common neighbors: Common neighbors is described as the amount of common neighbors shared by 2 objects

3. PropFlow [24]: a random walk-based measure PropFlow is suggested to determine the topological feature between 2 items. This specific approach assigns the weights to each and every path (with fixed length $l$ ) through the products of specifications of the flows on the edges.

We are able to observe that, almost all of the current topological features in homogeneous networks depend on neighbor sets or perhaps paths between 2 objects. Nevertheless, as there will be multi-typed objects as well as multi typed associations in heterogeneous networks, the neighbors of an object could belong to various types, and also the paths in between two objects could follow various meta paths and indicate various relations. Therefore, a more complex approach is needed to produce topological features in heterogeneous networks to differentiate paths with various meanings. In order to achieve this, the topology between the network objects should be defined by using the concept of meta-paths (covered earlier). The following measures are proposed on the meta paths determined topologies.

1. Path Count[37]: Path count measures the amount of path instances in between 2 objects following a certain metapath and it is denoted as $P C_{R}$ in which $R$ is the relation denoted through the meta path.

2. Random Walk: Random walk is a natural regularization of PropFlow (defined earlier) which is defined as: $R W_{R}\left(a_{i}, a_{j}\right)=\frac{P C_{R}\left(a_{i}, a_{j}\right)}{P C_{R}\left(a_{i}, \cdot\right)}$, where $P C_{R}\left(a_{i}, a_{j}\right)$ denotes the number of paths starting 
from $a_{i}$ following $R$ and ending with $a_{j}$ and $P C_{R}\left(a_{i}, \cdot\right)$ denotes the total number of paths starting with $a_{i}$ following $R$.

3. Degree-Weighted Path Count[13]: $D W P C$ is developed by adopting the existing method called PathPredict. DWPC downweights paths by high degree nodes when computing metapath prevalence. The strength of this method depends on parameter $w$ which is optimized to $w=0.4$, similar to Himmelstein et al [13] work. Every path gets a path degree product (PDP) calculated by:

(a) Extracting all meta edge-specific degrees on the path, $D_{\text {path }}$

(b) Raising each degree to $-w$ (damping exponent) which is optimized to 0.4 as mentioned earlier.

(c) Multiplying all of the exponentiated degrees to deliver the PDP

$$
P D P(\text { path })=\sum_{d \in D_{\text {path }}} d^{-w}
$$

By using the Path-degree Products summation, the Degree-weighted Path Count is obtained:

$$
D W P C_{m}(s, t)=\sum_{\text {path } \in \text { Paths }}(s, t) P D P(\text { path })
$$

For every meta path, we are able to apply some measure functions on it and receive a distinctive topological feature. For instance, Figure 2.3 is an example of how the feature between a user and a venue is calculated. The feature that is used in our model is the calculated degree-weighted path count (DWPC) between the users and the venues. For the defined metapaths, the users are treated as the source nodes and the venues are treated as the target nodes. As it is shown in Figure 2.3, between user "Branko" and venue "LS3", each metapath receives a different value of DWPC based on the path characteristics. 
metapath

paths

PDP

DWPC
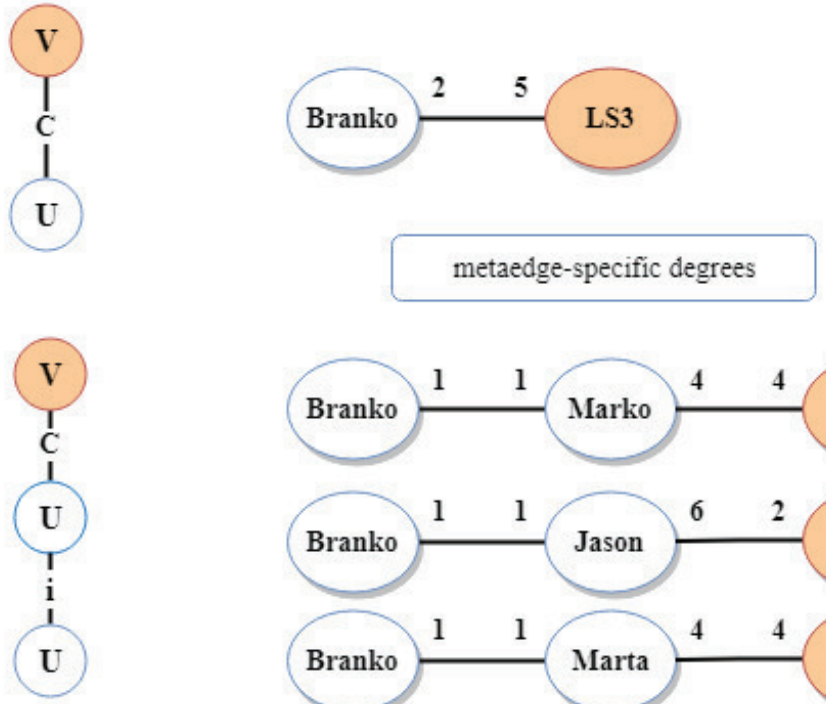

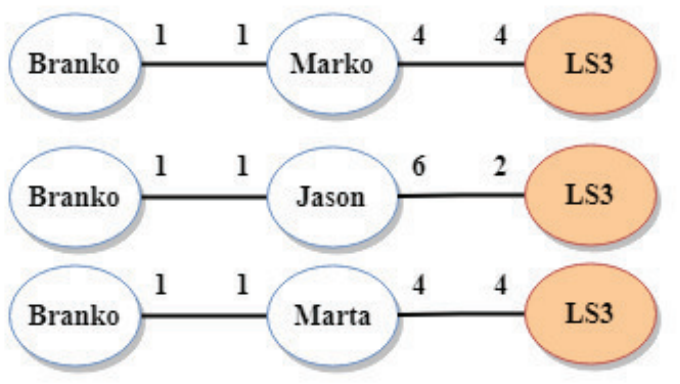

$$
\begin{gathered}
P D P(\text { path })= \\
\prod_{d \in D_{\text {path }}} d^{-w}
\end{gathered}
$$

0.316029

0.25

0.288

0.788

0.25

$D W P C($ metapath $)=$ $\sum_{\text {path } \in \text { Paths }} P D P($ path $)$

Figure 2.3: Degree-Weighted Path Counts in LBSNs

In addition to the meta-paths topological measures, similarity measures have been also a hot topic in the recent years. Similarity search continues to be thoroughly examined for traditional categorical as well as numerical data types in relational data. Most of these research studies are centered on homogeneous networks, such as Sim-Rank[14] and PageRank[15]. For example, in our work, a user may be interested in the (top-n) most similar point-of-interests for a given point-of-interest.

Nevertheless, these similarity methods overlook the subtlety of various types among links and objects. Using the new similarity measure of PathSim[40], the subtle semantics of similarity among peer objects of the Heterogeneous Information Network is captured.

Given a symmetric metapath $P$, the Meta path-based similarity measure (PathSim[40]) between the 
two nodes $\mathrm{x}$ and $\mathrm{y}$ is :

$$
s(x, y)=\frac{2 \times\left|\left\{p_{x \rightarrow y}: p_{x \rightarrow y} \in P\right\}\right|}{\left|\left\{p_{x \rightarrow x}: p_{x \rightarrow x} \in P\right\}+\left\{p_{y \rightarrow y}: p_{y \rightarrow y} \in P\right\}\right|}
$$

where $p_{a \rightarrow b}$ is a path instance between nodes $a$ and $b$.

PathSim is able to identify objects that are not only strongly connected but additionally share identical visibility in the network considering the meta path. The intuition behind it is that 2 similar peer objects should not solely be strongly linked, but additionally share comparable visibility. This similarity measure has been used in our Ensembled Point-of-Interest Recommendation as a feature of feature-based matrix factorization which will be discussed in Chapter 3 .

\subsection{Feature-Based Matrix Factorization}

Since early 1990s, research on recommender methods has shown excellent efficiency in solving the issue of information overload by connecting information actively, instead of passively, to possibly associated people based on their information utilization history $[1,21]$. Recommendation plays highly crucial role in our daily lives. Recommender systems automatically recommend to a user things that could be appealing to her. Recommender system $(R S)$ origins date back to many similar research disciplines, for example cognitive science, approximation principles as well as information retrieval. The primary task is figuring out beneficial patterns describing the connection between users and items from user item usage history, and after that to generate predictions for potential user-item links based on these patterns [27].

\subsubsection{Collaborative Filtering Based Approaches}

In general, there are two variants of recommendation approaches: collaborative and content-based, $\mathrm{CF}$ and $\mathrm{CB}$, filtering based approaches. $\mathrm{CB}$ filtering is popular for recommendation systems design, and uses the information of products to produce features and also attributes to match up with user profiles. The CF approach is considered the most preferred method for recommendation systems design. It uses 
a significant volume of data collected from user actions in the past and predicts what items users will be interested in future. It does not need to evaluate the content of the items. Rather, it depends on the relationship between items and users, which are generally encoded in a rating feedback matrix with each component representing a certain user rating on a certain product. An illustration of the Collaborative Filtering process is shown in Figure 2.4.

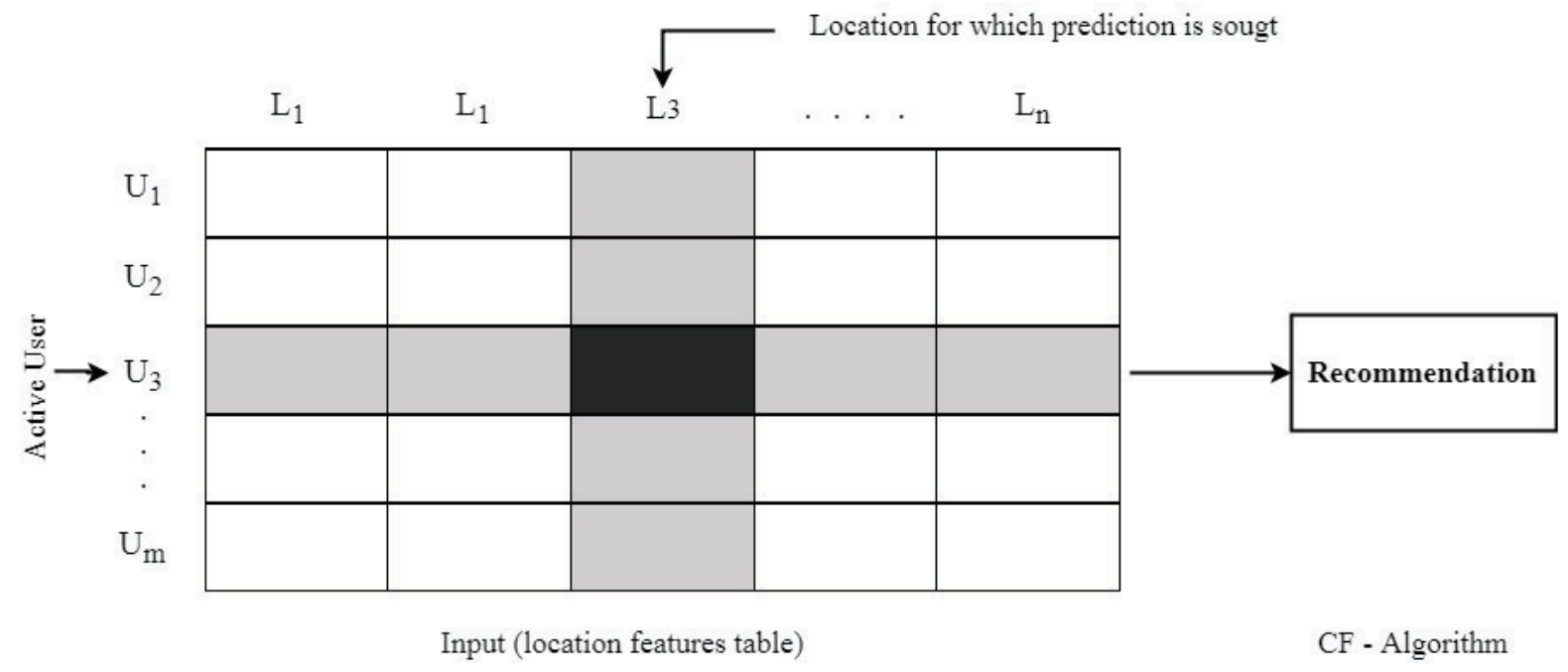

Figure 2.4: Collaborative Filtering Process

The common $\mathrm{CF}$ recommendation task is predicting the missing ratings by provided users or for specified items by data exploring and mining the user item score matrix [42]. For instance, Point-ofInterest recommendation using $\mathrm{CF}$ models can be seen in Fig 2.4 where CF algorithms signify entire $m \times n$ user location statistics as being a visits matrix, RM. Each entry in RM belongs to the amount of visits of the ith user on the jth location. Each person check-ins is inside a numerical scale and it can also be 0 indicating the user has not visited that location. However, as discussed in the following chapter, this is not how we deal with this problem since this is a solution cannot deal with the sparse RM matrix in POI Recommendation. 


\subsubsection{Matrix Factorization Based Procedures}

Matrix-factorization (MF) based procedures have found to be scalable and accurate highly in dealing with collaborative filtering $(\mathrm{CF})$ conditions. The CF is generally implemented by a handful of approaches. The latest advances in this particular area show that matrix-factorization (MF)-based models are scalable and highly accurate for many cases $[17,51,44,34]$. An MF based model operates by mapping both the items along with users directly into the same latent feature space, training preferred user/item features on existing ratings, and then creating predictions for unidentified ratings highly depending on the internal products of related user item feature-vector pairs. Due to the typical low-rank of the ratingmatrix created by its extreme sparsity, the dimension of the latent feature space could be set to a low value without impairing accuracy.

Thus, the dimensions of feature matrices in a MF based design is linearly associated with item and user counts. Many MF based methods have been proposed and effectively applied to CF recommenders. For instance, at the Netflix-prize competition, the Collaborative Filtering recommender which was based on regularized Matrix-Factorization model has established high performance [10]. The concept of MF based CF has additionally been employed in several relevant areas, e.g., image tagging in image processing [53] and video re-indexing in information retrieval [43].

The typical framework of matrix factorization approach in the recent studies is singular value decomposition based recommender [4] which is the earliest model of its kind. In order to execute a regular SVD process, this particular model demands prefilling the unidentified reviews synthetically with the rating data. The SVD based model has a benefit in storage effectiveness, which is crucial for the majority of industrial applications. 


\section{$2.3 \quad$ Neural Embeddings}

Neural embedding approaches, such as word2vec, proposed in [19] for word embedding learning is often put into use to model numerous kinds of sequential data. The core concept could be summarised as follows. Provided a set of symbolic representation sequences, a fixed-length vector representation for every symbol could be learned in a latent space by exploiting the context information amongst symbols, in which regularly "similar" symbols will likely be mapped to close by positions [52]. If we treat every single location ID as a word token, and also turn the historical location visit records of a user to a timestamped sequence, we are able to subsequently make use of the exact same methods to acquire location embeddings.

Skip-gram model vs. Continuous Bag-of-Words: Mikolov et al.[28], proposed two recurrent neural architectures for training product embeddings, called Continuous Bag-Of-Words (CBOW) and the Skip-gram model which has been used in our work. The key distinction between these two architectures lies in the path of prediction: CBOW predicts the present item using the surrounding context, while Skip gram predicts the context together with the present token as also shown in Fig 2.5.

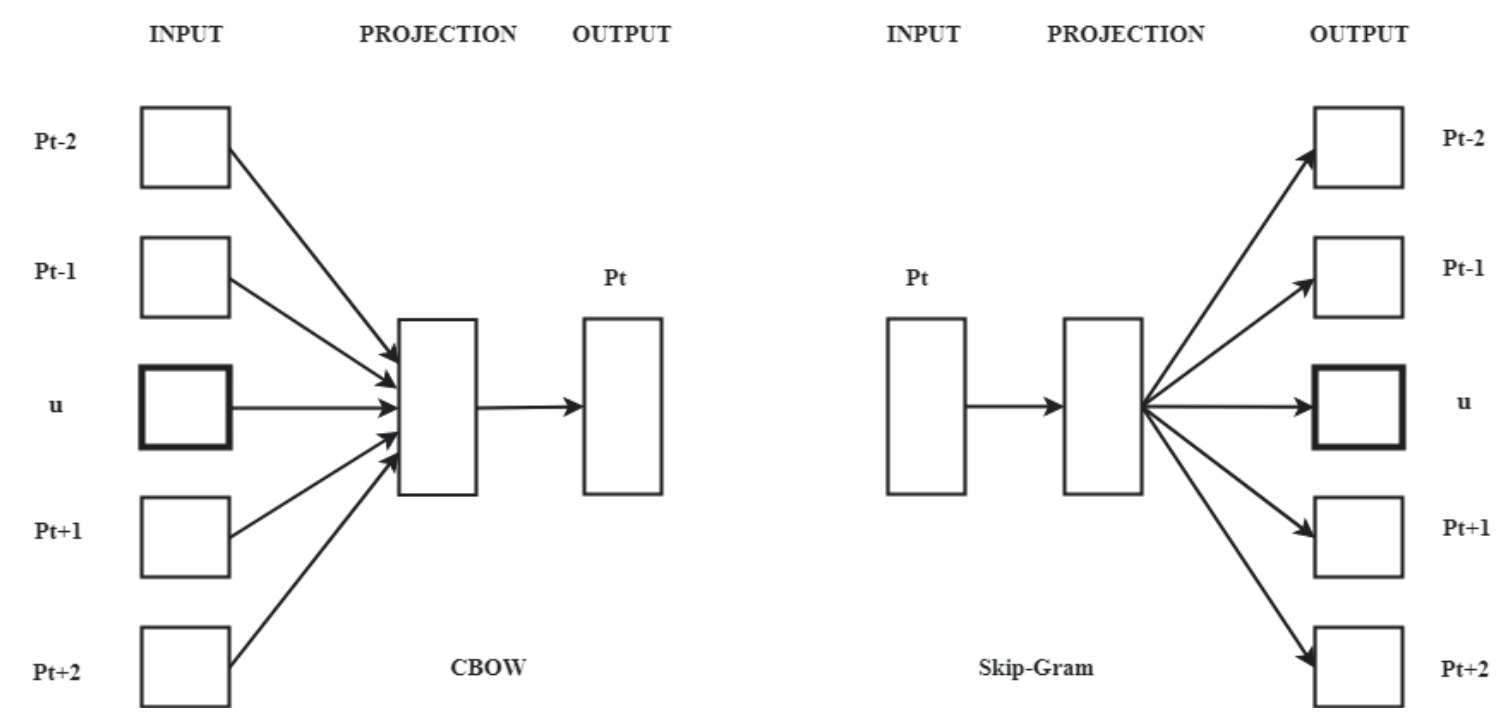

Figure 2.5: Recurrent neural architectures 


\subsection{Point-of-Interest Recommendation}

In this section, we first discuss pure check-in data based POI recommendation methods. Next, we split POI recommendation procedures into state based POI recommendation, social impact and temporal and spatial impact influenced depending on which type of supplemental information are merged with check-in information to enhance the quality of POI recommendation.

\subsubsection{Pure Check-in Data Based Point-of-Interest Recommendation}

Generally there has been strong work on state-based Point-of-Interest (venue) suggestion within the literature. Gambs et al.[8] have suggested a version of typical Markov Chains that includes the n previous visited venues of users. In this work, the next place of the users is predicted by using the mobility Markov chain which was developed for every individual user. For instance, if user "Lionel" has visited "Tim Hortons" at $t=0$, "Cineplex Empress Walk" at $t=1$ and "Tim Hortons" at $t=2$, these models (based on their $n$ value) will recommend one of the locations at time 0 or 1 for the next place of the user. The majority of the location recommendation Markov based models just use the geographical information of the locations without taking into consideration the context of the user footprints.

Supervised learning models were additionally examined in [30] for location recommendation. In this particular work, two supervised procedures, linear M5 and regression trees have been compared and it was discovered that M5 trees attain higher prediction accuracy. Much like various other works, the set of attributes that have been used for training in the classifiers had been the user transitions between places as well as spatio temporal features of the check-in footprints. In our work, we've trained our model with the linear regression classifier and also have accomplished much better recommendation accuracy as a result of using the features collaboratively rather than independently as compared to the work by Noulas

et al[30]. In [47] and also [26], the significance of venue context information like different categories for location recommendation had also been investigated. 
The accuracy of point-of-interest recommendations was elevated by getting the users' preferences which were depending on the place categories they had visited. While in many established work the current place of the user is necessary for location recommendation; in our work, we clearly show increased performance as compared to the state of the art without needing information regarding the last place of the user.

Because of the success of Netflix Prize tournament, matrix factorization [18] based recommendation algorithms have received a substantial popularity due to their efficiency and effectiveness in dealing with very large user item rating matrix. It is intuitive that matrix factorization strategies can certainly be put into use for POI recommendation in LBSNs and it can be commonly used in POI recommendation by treating locations as items. In [6], matrix factorization is fused with geographical effect by using Multi center Gaussian Model (MGM) and social influence. Throughout the work [4], location recommendation is based on the likelihood of a Gaussian distribution model, which is applied to the checked in location centers together with the fusion framework with user preferences. Nevertheless, the information of the check-in footprints for instance the geographical context aren't taken into consideration.

In addition, the authors in [48] suggested item-based and user-based POI recommendation methods. In their work, item based POI recommendation technique assumes that users are attracted to quite similar POIs. However, User-based POI suggestion approach assumes that quite similar users have equivalent tastes for locations and tends to make POI recommendations based on the views of almost all very similar neighbors. 


\subsubsection{Social Impact}

Collaborative recommendation approaches have been extensively explored in traditional recommender systems due to its positive impact on the quality of recommendations. Inspired from the presumption that friends of LBSNs share more common pursuits than non friends, several POI recommendation strategies enhance the quality of their recommendations by taking community impact straight into account such as [48]. For instance, in their approach, POI recommendations is based on a friend-based collaborative filtering model. Their model is trained based on the friendships similar preferences instead of the similarity between all the users in the social network. However, in [6] recommendations are made based on social influence of the network being examined. In addition, we have taken the social impact based on the friendship between users in our Heterogeneous Link Prediction POI recommendation model which will be covered in the next chapter.

\subsubsection{Temporal and Spatial Impact}

Social and temporal influence on the quality of Point-of-Interest recommendations has been well studied in the past few years. Yuan et al. [50] believe that users tent to check out locations that are different at different time and proposed time aware POI recommendation algorithm. Specifically, their proposed POI recommendation algorithm expands the user-based POI recommendation algorithms by leveraging the time aspect when computing the similarity between two individuals along with taking into consideration the historic check-in times to make POI recommendation. For instance, Yang et al.[47] have recommended a fusion framework, which exploits both equally temporal and spatial activity personal preferences (using tensor factorization) of users to predict the next point-of-interest. For every person, the spatial features are captured by generating Personal Functional Regions, that are made based on frequented regions which the person visits. This particular design utilizes the temporal and spatial features separately for recommendation to users. In this work, each region is assigned to a category which 
the user is much more keen on depending on her historical visits.

As a result, if the user is close to each region, the category assigned to that particular region is put into use for venue recommendation. Additionally, in this particular work, the lifestyle actions of users is noticed by using the temporal nature of the check-ins. It must be noted that Yang's model doesn't recommend venues to users and mainly recommends location categories.

Nevertheless, the rich information of the check-in data for instance the spatial context aren't taken into consideration. Recently, Pham et al.[32] proposed an algorithm which exploits the spatial impact among venues, rather than dealing with them separately to evaluate attractiveness. Conversely, there are actually work which exploit the transition of users across venues along with solely directly predicting users' interest in venues. For instance, Noulas et al. [30] proposed a set of features which exploit information on transitions among kinds of venues, mobility moves in between venues and spatio temporal qualities of user check-in patterns.

In order to integrate a pair of user along with venue associated information, Xie et al.[45] proposed a graph based embedding that captures sequential impact, geographical impact, temporal cyclic as well as semantic influences in a single approach to produce a spatio-temporal context awareness model to tracks user's most recent preferences. Guo et al.[11] viewed the venue recommendation issue as being a node ranking issue that operates on a heterogeneous graph.

\subsubsection{Summary}

In this section, we covered a detailed study of Link Prediction, Feature-Based Matrix Factorization approaches and recent state-of-art Point-of-Interest models. Explicitly, we have divided Point-of-Interest models into three categories which are 1) Pure Check-in Data based POI Recommendation models, 2) Collaborative-based approaches and 3) Spatial and Temporal based POI recommendation models. The details behind using these approaches for the problem of POI recommendation is covered in Chapter 3. 


\section{Chapter 3}

\section{The Proposed Approach}

The objective of our work is to recommend a point-of-interest (venue) to a user based on her historical check-in data. We propose turning the problem of venue recommendation into feature based recommendation. The primary goals of our work are i) find the rich features from user check-ins and generate a new set of features for point-of-interest recommendation; and ii) compare the results of the recommendations made by comparing the best performing features with each other and use the best ones for the problem of POI recommendation.

As it can be seen in Fig 3.1, we first use the checkins from our dataset and build two models: i) Heterogeneous Information Network from the user footprints features (Users, Venues, Categories of Venues and the Regions of Venues) which will be covered in the next section; ii) Develop a new set of features derived based on the neural embedding representation of venues and users. Depending on the curated training set, we measure the level of influence of every feature on the overall performance as well as robustness of the point-of-interest recommendation results. We find the top-n similar users and venues by using different methods in each model. For Heterogeneous Link Prediction, we use the idea of PathSim (covered in Section 2.2) and its' meta-path based similarity search to find the similar users and venues. Likewise, we find the top-k similar users and venues in the Neural Embedding approach 
based on the cosine similarity between users and venues embedding vectors.

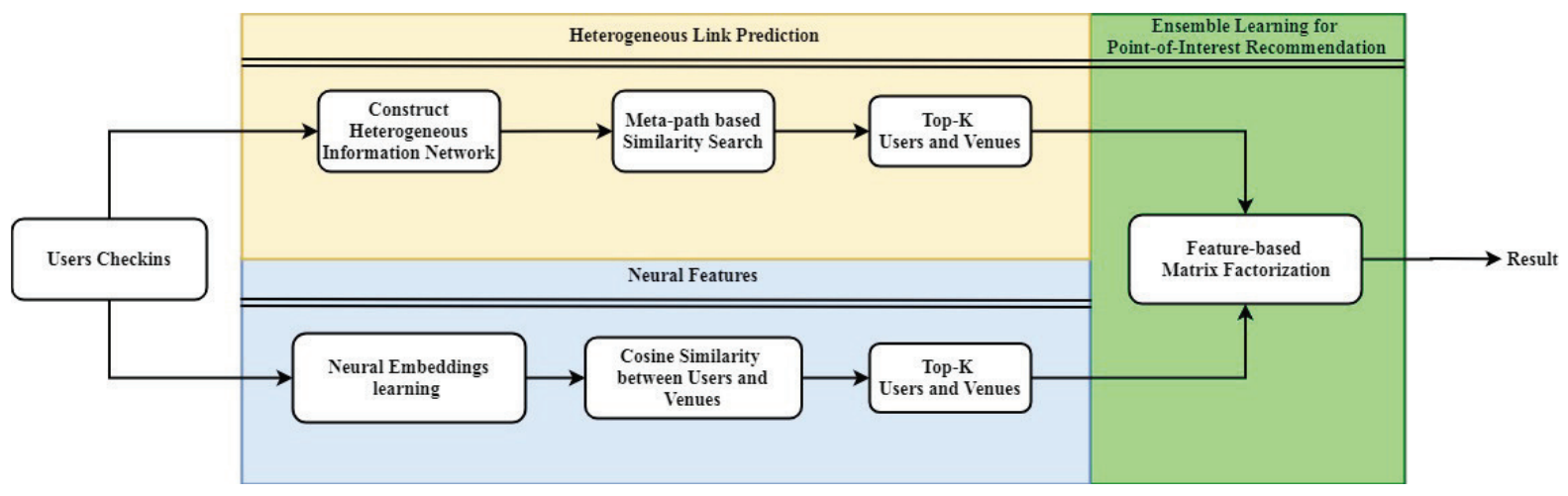

Figure 3.1: Approach Overview

We show the specifics of every step in the following subsections.

\subsection{Heterogeneous Link Prediction}

The objective of our work is to recommend a point-of-interest (venue) to a user based on her historical check-in data. Formally, given the check-in profile of a user in time interval $t$ (Definition 1), we aim at recommending a list of venues that the user may be interested in at time interval $t+1$.

Definition 1. (User Check-in Profile). The check-in profile of user $u \in \mathbb{U}$ at time interval $t$, with respect to a set of venues $\mathbb{V}$, denoted by $C P^{t}(u)$, is represented by a vector of weights over the $K$ venues, i.e., $\left(f_{u}^{t}\left(v_{1}\right), \ldots, f_{u}^{t}\left(v_{K}\right)\right)$, where $f_{u}^{t}\left(v_{k}\right)$ is equal to one if user $u$ checked in at venue $v$ in time interval $t$ and 0 otherwise.

We propose to turn the problem of venue recommendation into a link prediction problem that operates over a heterogeneous graph. In addition to historical check-in data of users, there are other types of data that can be considered while recommending venues, namely venue categories, venue regions and 
user relationships. In this paper, we combine these data points into a unified heterogeneous graph representation model to consider them simultaneously. An illustration of our underlying representation model G can be found in Fig 3.2.

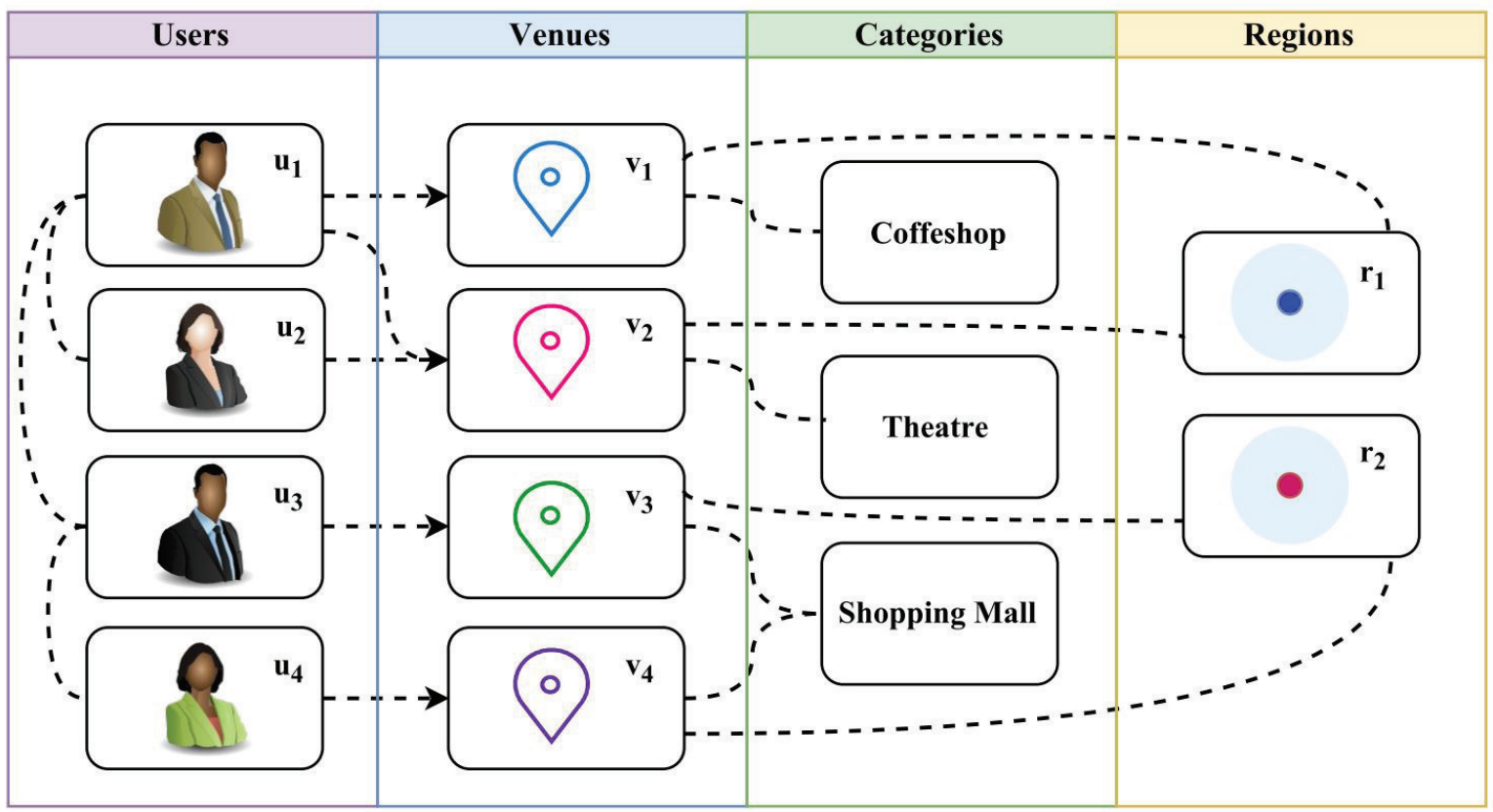

Figure 3.2: Sample heterogeneous graph representation of LBSN data.

As illustrated in Fig 3.2, our representation model contains four types of nodes and four types of relations. Besides User and Venue nodes and User-Venue relations that represent historical check-in data of users, other types of data that are included consist of the following:

- Category nodes: In location-based social networks, venues are organized by a hierarchical category tree which provides a semantic classification of the various venues. For example, Foursquare contains a 3-level category hierarchy where categories are grouped into 10 top-level categories, such as Event, Food, Nightlife Spot and Residence. Each top-level category is classified into different subcategories. In our approach, we have infused the categories at the lowest level (Level 
3) as our category nodes.

- Region nodes: Each region indicates a geographical area. Given the longitude and latitude of existing venues, we use X-means clustering [31] to cluster geographical coordinates and extract different regions.

- Venue-Category relations: Based on the hierarchical category tree defined in LBSNs for organizing venues, we assign each venue to its corresponding category in the lowest level of the hierarchy.

- Venue-Region relation: To identify the region of a venue, we calculate the Euclidean distance between the venue geographical coordinate (longitude and latitude) and the center of the identified regions and connect each venue to its nearest region.

- User-User relation: Users are connected to each other based on the friendship relation among them on the LBSN. By using this relation, potential interaction between users is also taken into account for point-of-interest recommendation.

Having built the representation model $G$, in order to recommend a point-of-interest to a user $u \in \mathbb{U}$, we formulate a graph-based link prediction problem that operates over $G$. As our representation model is a heterogeneous graph, the neighbors of an object could belong to multiple types and the paths between two objects could have different meanings. Therefore, it is not possible to apply link prediction strategies such as Adamic/Adar and Common Neighbor, which treat all types of nodes and relations as the same in the form of a homogeneous graph [22].

Sun et al. [40] proposed the concept of heterogeneous information networks and the meta-path concept for heterogeneous information network analysis, which are now widely known and used in different data mining tasks such as ranking [25], clustering [36], link prediction [3], and influence analysis [35]. In order to solve the problem of link prediction in heterogeneous graphs, Sun et al. [37] proposed the PathPredict method, i.e., meta path-based relationship prediction model to predict links between dissimilar node types. Therefore, to distinguish different types of objects and relations, following the works 
in $[37,39]$, we use the PathPredict method to determine the relevance of a point-of-interest $v \in \mathbb{V}$ for a user $u$. The core of the PathPredict method rests on the idea of meta-paths. A meta-path is a path defined over the heterogeneous network schema which can be used to define topological features with different semantic meanings. Fig 3.3 summarizes our representation model using a meta-structure known as the network schema.

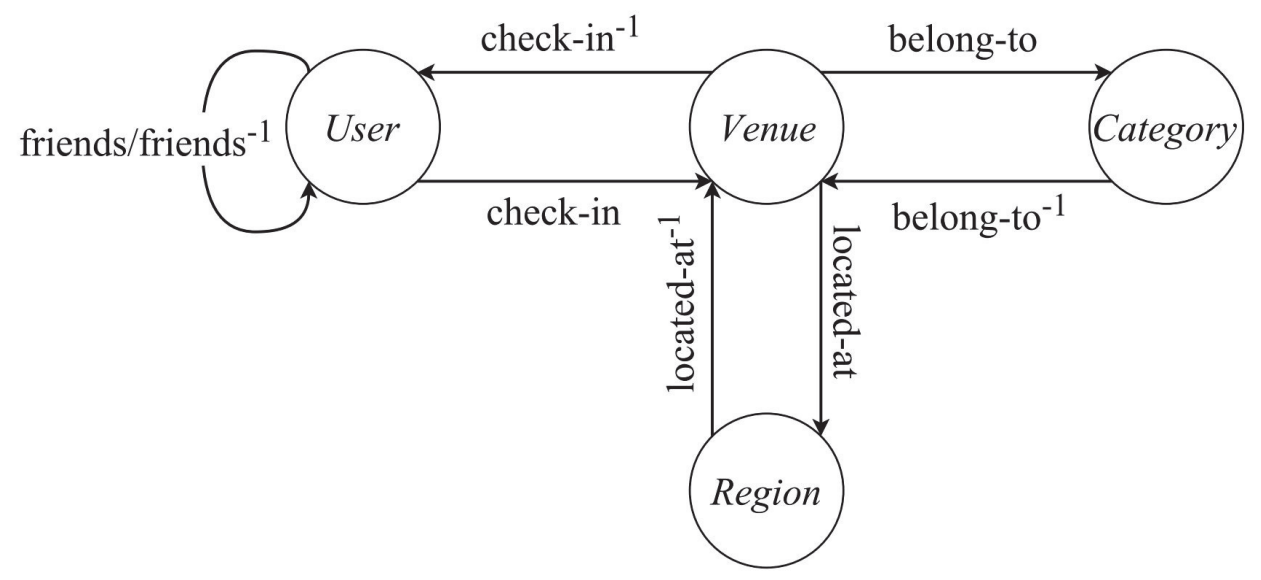

Figure 3.3: Network schema of the representation mode.

Based on PathPredict, for the target relation $\langle\mathbb{U}, \mathbb{V}\rangle$, we define a set of meta-paths starting with type $\mathbb{U}$ and ending with type $\mathbb{V}$ other than the target relation itself. We extract all such meta-paths by traversing the network schema using Breadth-First Search (BFS) within a fixed length constraint (max $=3$ ). The extracted meta-paths and their semantic meaning are shown in Table 3.1. For example, The meta-path $\mathbb{U}-\mathbb{V}-\mathbb{C}-\mathbb{V}$, i.e., user-venue-category-venue considers those venues which belong to the same category of the historical check-in venues of a user as her next check-in venue. 


\begin{tabular}{|c|c|}
\hline Meta-path & Meaning of the Metapath \\
\hline $\mathbb{U}-\mathbb{U}-\mathbb{V}$ & A user visits a venue where her friends have visited \\
\hline $\mathbb{U}-\mathbb{V}-\mathbb{C}-\mathbb{V}$ & A user visits venues that belong to the same category \\
\hline $\mathbb{U}-\mathbb{V}-\mathbb{R}-\mathbb{V}$ & A user visits venues located in the same region \\
\hline
\end{tabular}

Table 3.1: Meta-Paths between users and venues

Once the meta-paths are retrieved from the network schema, for each user-venue pair in the representation model $G$, we use the Degree-Weighted Path Count metric [13] to quantify each meta-path as a topological feature in the training step. For the given meta-path, Degree-Weighted Path Count penalizes paths, which pass through high-degree nodes. Then, given all user-venue pairs in the representation graph $G$ and the extracted topological features for them, a logistic regression classifier is trained as the learning model to recommend a ranked list of points-of-interest for a given user.

\subsection{Neural Embedding Model}

Similar to the previous model, the objective of our work is to recommend a point-of-interest (venue) to a user based on her historical check-in data. We formally define the point-of-interest recommendation problem as follows:

Definition 2. (Venue Recommendation). Let the check-in sequence of user $u \in \mathbb{U}$, denoted by $C S(u)=\left(v_{u}^{t}\right)$, be a sequence of venues ordered on $t$, where $v_{u}^{t}$ shows a venue $v \in \mathbb{V}$ that user $u$ checked in at time t. Based on the CS $(u)$ from $T$ consecutive time intervals, we aim at recommending a ranked list of venues that the user $u$ may be interested in, each denoted by $v_{u}^{x}$ such that $x \in T+1$. The recommendations are ranked descendingly, based on the predicted degree of interest of user $u$ in $v$ at time $x$.

Our key contribution is to learn user and venue representations in the same embedding space based 
on the check-in sequence of users with the expectation that a user would be closer to the venues that she has checked-in at compared to those she has not, in the vector space. We show that by embedding users and venues in the same space, it will be possible to construct different types of features based on these embeddings, which can be directly incorporated into a feature-based recommendation model.

\subsubsection{User and Venue Embeddings}

Our goal is to learn user and venue embeddings in the same space so that a user is closer to the venues that she has checked-in compared to those that she has not. To this end, given the check-in sequence of all the users up to time interval $T$, i.e., $\left\{C S(u)=\left(v_{u}^{t}\right) \mid u \in \mathbb{U}\right\}$, we map each user and venue into an $L$-dimensional embedding vector, denoted by $e_{u}$ and $e_{v}$ within the same embedding space in the same feature space by applying a neural embedding model based on the framework shown in Figure 3.4.

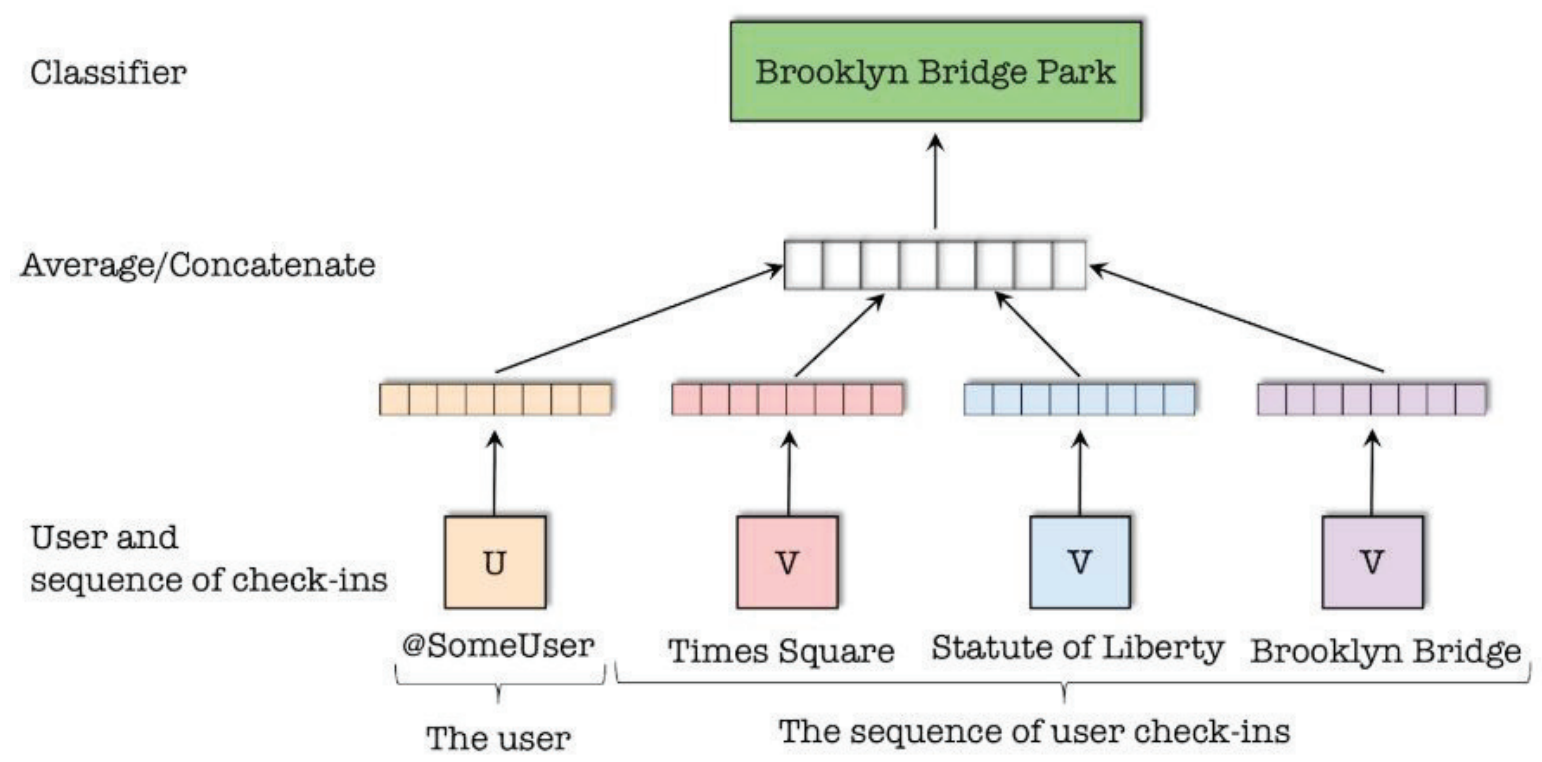

Figure 3.4: A framework for learning user and venue embeddings in the same feature space.

We consider the sequence of checked-in venues of each user $u$ as a sentence and place the ID of user 
$u$ at the beginning of each sentence which results in $|\mathbb{U}|$ sentences. In the learning process, both the ID of users $|\mathbb{U}|$ and venues $|\mathbb{V}|$ are treated as word tokens and a user ID is essentially always associated with a set of check-ins. In other words, during training, for each sentence, the sliding context window will always include the first word in the sentence (i.e., user ID). Formally, given the check-in sequence of each user $u, C S(u)=\left(v_{u}^{t}\right)$, and a context window size of $k$, the objective is to maximize the following log probability:

$$
\frac{1}{T} \sum_{t=k}^{T-k} \log \operatorname{pr}\left(v_{u}^{t} \mid v_{u}^{t-k}, \ldots, v_{u}^{t+k}, u\right)
$$

We adopt the skip-gram model for which the conditional prediction probability is characterized by a softmax function as follows:

$$
\begin{gathered}
\operatorname{pr}\left(v_{u}^{t} \mid v_{u}^{t-k}, \ldots, v_{u}^{t+k}, u\right)=\frac{\exp \left(e_{\text {context }}^{\top} \cdot e_{v_{u}^{t}}\right)}{\sum_{v \in \mathbb{V}} \exp \left(e_{\text {context }}^{\top} \cdot e_{v_{u}^{t}}\right)} \\
e_{\text {context }}=h\left(e_{v_{u}^{t-k}}, \ldots, e_{v_{u}^{t+k}}, e_{u}\right)
\end{gathered}
$$

Here, $e_{v_{u}^{t}}$ denotes the latent embedding vector of venue $v_{u}^{t} \in C S(u)$ and $e_{\text {context }}$ denotes the associated context vector, which is obtained by averaging over the embedding vectors of the context information (Equation 3.3). Further, $h($.$) is a function that averages the embedding vectors. By sampling a target$ venue and its context window at each iteration, our neural network is trained using stochastic gradient descent and updates the parameters gradually via backpropagation. As such, the final result of the learning process is an $L$-dimensional embedding for each user $u \in \mathbb{U}$ and each venue $v \in \mathbb{V}$, i.e. $e_{u}$ and $e_{v}$, respectively. 


\subsubsection{Embedding-based Features}

Given user and venue embeddings, i.e., $e_{u}$ and $e_{v}$, which are located in the same embedding space, we aim at exploiting these vectors to define a set of features for the purpose of venue recommendation. These features are categorized into three categories:

User features: for each user $u \in \mathbb{U}$, we propose two types of features: 1) user embedding feature, which is equivalent to the $L$-dimensional embedding vector for user $u$, i.e. $e_{u}$ : and 2) top- $N$ similar users feature that is inspired from the main idea of collaborative filtering based on which similar users share similar check-in behaviour in the future. Therefore, for a given user $u$, we consider her top- $N$ similar users, denoted by $S_{u}$. This provides us with $N$ features, which correspond to the most similar users to a target user. To compute the similarity of two users $u_{1}$ and $u_{2}$, we apply cosine similarity between $e_{u_{1}}$ and $e_{u_{2}}$.

Venue features: for each venue $v \in \mathbb{V}$, we define two types of features: 1) venue embedding feature, which is the learnt $L$-dimensional embedding vector for venue $v$, i.e., $e_{v}$; and 2) top- $N$ similar venues feature where for a venue $v$, we calculate its similarity with other venues through cosine similarity of their embedding vectors and then select the top- $N$ most similar venues to $v$, denoted as $S_{v}$.

Global user-venue interaction feature: Given both user and venue embeddings in the same feature space, we can incorporate a global feature to denote the similarity between any given user and venue pair. The global feature of a user-venue pair such as $(u, v)$ is calculated by the cosine similarity between $e_{u}$ and $e_{v}$. 


\subsubsection{Venue Recommendation}

Here, we show how the proposed features can be encoded into a feature-based matrix factorization model as follows:

$$
\widehat{r}_{u, v}(\alpha, \beta, \gamma)=\mu+\sum_{j} b_{j}^{(g)} \gamma_{j}+\sum_{j} b_{j}^{(u)} \alpha_{j}+\sum_{j} b_{j}^{(v)} \beta_{j}+\left(\sum_{j} \alpha_{j} x_{j}\right)^{\top}\left(\sum_{j} \beta_{j} y_{j}\right)
$$

where $\alpha \in R^{N_{\alpha}}, \beta \in R^{N_{\beta}}$ and $\gamma \in R^{N_{\gamma}}$ are the input vectors consisting of the features of user $\mathrm{u}$, the features of venue $\mathrm{v}$ and the global feature for the pair $(u, v)$ with the lengths of $N_{\alpha}, N_{\beta}$ and $N_{\gamma}$, respectively. Further, $b_{j}^{(g)}, b_{j}^{(u)}$ and $b_{j}^{(v)}$ are the global, user and venue bias parameters. The latent vectors $x_{j}$ and $y_{j}$ capture the $j^{t h}$ user feature and the $j^{t h}$ venue feature, respectively. The global features and bias values do not have any corresponding latent vectors. The response value, i.e., $\widehat{r}_{u, v}(\alpha, \beta, \gamma)$, predicts whether user $u$ will check-in at venue $v$ in the future. In the following, we show how to encode our features into Equation 3.4.

Encoding user features: In the user input vector $\alpha \in R^{N_{\alpha}}$, consisting of the user features of user $\mathrm{u}$, we reserve the first $|\mathbb{U}|$ dimensions to one-hot encode the ID of user $\mathrm{u}$ and its top- $\mathrm{N}$ most similar users, i.e. $S_{u}$. Therefore, given $Q=\{u\} \cup S_{u}$, each user $q \in Q$ is encoded by inserting a "1" in the $q^{t h}$ dimension of the $|\mathbb{U}|$-dimensional vector. The rest of dimensions are set to "0". Then, given a user u, we use the dimensions from $(|\mathbb{U}+1|)$ to $(|\mathbb{U}+L|)$ to encode her user embeddings, $e_{u}$. Therefore, the total number of user features is $N_{\alpha}=|\mathbb{U}|+L$.

Encoding venue features: We reserve the first $|\mathbb{V}|$ dimensions in the venue input vector $\beta \in R^{N_{\beta}}$, to one-hot encode the venue $v$ and its top-N most similar venues, i.e. $S_{v}$. Then, given a venue $v$, we use the dimensions from $(|\mathbb{V}+1|)$ to $(|\mathbb{V}+L|)$ to encode the venue embeddings, $e_{v}$. The total number of venue features is $N_{\beta}=|\mathbb{V}|+L$.

Encoding global user-venue interaction feature: The idea behind the user-venue interaction fea- 
ture is that a user is more likely to check-in at a venue closer in the latent feature space to those she checked in at before; therefore, the corresponding venue should receive a larger global bias value. Therefore, we define the global user-venue interaction feature as $\gamma_{1}=\operatorname{cosineSim}\left(e_{u}, e_{v}\right)$ and the number of global features is $N_{\gamma}=1$.

With these coded features, for a user-venue pair $(u, v)$, based on Equation 3.4, we have the following factorization formula:

$$
\begin{aligned}
& \widehat{r}_{u, v}(\alpha, \beta, \gamma)=\mu+b_{1}^{(g)} \gamma_{1}+\sum_{j} b_{j}^{(u)} \alpha_{j}+\sum_{j} b_{j}^{(v)} \beta_{j}+ \\
& \left(\sum_{q \in\{u\} \cup S_{u}} x_{q}+\sum_{l=1}^{L} e_{u, l} x_{|\mathbb{U}|+l}\right)^{\top}\left(\sum_{p \in\{v\} \cup S_{v}} y_{p}+\sum_{l=1}^{L} e_{v, l} y_{|\mathbb{V}|+l}\right)
\end{aligned}
$$

The parameters are trained by minimizing the log-likelihood loss function using stochastic gradient descent. Based on Definition 1, our goal is to construct a ranked list of venues that $u$ may be interested in, at time interval $\mathrm{T}+1$. Therefore, for a given user $u$, we first calculate the value $\widehat{r}_{u, v}$ for all the pairs $\{(u, v) \mid v \in \mathbb{V}\}$ and then construct the ranked list by descendingly ranking on $\widehat{r}_{u, v}$.

\subsection{Ensemble Features}

As stated previously, the objective of our work is to come up with a solution for Point-of-Interest Recommendation. As discussed earlier, we have proposed two methods for this specific problem, heterogeneous link prediction along with neural embedding. Similar to our second proposed approach of using neural embeddings for point-of-interest recommendation problem, in this section we propose to turn the problem of venue recommendation into feature-based recommendation.

Hence, the data that is used in the feature-based matrix factorization model consists of several sets 
of features. As mentioned earlier, inspired from the principal idea of collaborative filtering technique based upon which identical users share identical check-in behaviour as time goes by, we have proposed two features of top- $N$ meta path-based similar Users and Venues (equivalent to top- $N$ similar users and top- $N$ similar venues). Since, meta path is a path consisting of a sequence of associations determined between various object types, a meta-path based unique similarity measure called PathSim[40] is a better solution then applying cosine similarity on the meta-path topology metrics such as DWPC, RW, among others. to find the similarity between nodes.

Unlike path count and random walk-based similarity measure, the intuition behind PathSim is that two similar peer objects should not merely be strongly connected, but additionally share comparable visibility. The problem of Point-of-Interest recommendation in this model is solved similarly to the Neural Embedding Model. There are various features used in this model's feature-based matrix factorization for the problem of Point-of-Interest recommendation:

(i) Embeddings Feature: User and venue embeddings, i.e., $e_{u}$ and $e_{v}$

(ii) Top-N Similar Users and Venues Feature

(a) Embedding Similar Users and Venues: Computed by using cosine similarity between their embedding vectors.

(b) Meta Path-based similar Users and Venues: Gathered by using similarity search framework of PathSim between Users and Venues.

\section{(iii) Global User-Venue Interaction:}

(a) Embedding-based Global Feature: Computed by cosine similarity between the user eu and venue embeddings ev pairs $(\mathrm{u}, \mathrm{v})$.

(b) Metapath-based Global Feature: Computed by using the PathSim metapath based similarity search in user-venue pairs. 
Since, PathSim is a metapath based similarity framework which finds two similar peer objects in the heterogeneous information network, a set of meta-paths are defined for finding similarities between Users, Venues and Users-Venues. Based on the PathSim approach, we need three target relations to find similarities for the nodes (Users and Venues) that we are dealing with for the problem of Point-of-Interest recommendation. For user similarities the target relation is $<\mathbb{U}, \mathbb{U}\rangle$, venue similarities $<\mathbb{L}, \mathbb{L}>$ and user-venues similarities $\langle\mathbb{U}, \mathbb{L}>$. The user-venue similarity metapaths are similar to the ones discussed in the Heterogenous Link Prediction model. We extract all such meta-paths by traversing the network schema using Breadth-First Search (BFS) within a fixed length constraint ( $\max =3)$. The extracted meta-paths and their semantic meaning are shown in the following tables.

\begin{tabular}{|c|l|}
\hline Meta-path & \multicolumn{1}{c|}{ Meaning of the Meta-path } \\
\hline $\mathbb{U}-\mathbb{U}$ & Similarity of users based on their friendship \\
\hline $\mathbb{U}-\mathbb{L}-\mathbb{U}$ & Similarity of users based on the venues they have both visited \\
\hline $\mathbb{U}-\mathbb{L}-\mathbb{R}-\mathbb{L}-\mathbb{U}$ & Similarity of users based on the venues they have both visited in the same region \\
\hline $\mathbb{U}-\mathbb{L}-\mathbb{C}-\mathbb{L}-\mathbb{U}$ & Similarity of users based on the venues they have both visited in the same category \\
\hline
\end{tabular}

Table 3.2: PathSim Meta-Paths between Users

\begin{tabular}{|c|c|}
\hline Meta-path & Meaning of the Meta-path \\
\hline $\mathbb{L}-\mathbb{U}-\mathbb{L}$ & Similarity of locations visited by the same user \\
\hline $\mathbb{L}-\mathbb{R}-\mathbb{L}$ & Similarity of locations in the same region \\
\hline $\mathbb{L}-\mathbb{C}-\mathbb{L}$ & Similarity of locations in the same category \\
\hline $\mathbb{L}-\mathbb{U}-\mathbb{U}-\mathbb{L}$ & Similarity of locations based on the users friends checkins \\
\hline
\end{tabular}

Table 3.3: PathSim Meta-Paths between Venues.

Similar to our previous proposed approach, we have encoded the defined features into a featurebased matrix factorization model. We have formulated a feature based matrix factorization design for 
the process of Point-of-Interest Recommendation similar to (3.4). 


\section{Chapter 4}

\section{Empirical Evaluations}

We conduct experiments to show our proposed is more accurate compared to the state-of-the-art. We discuss the dataset used, baselines, gold standard, and metrics we adopt to examine our proposed models.

\subsection{Heterogeneous Link Prediction Method}

In this section, we describe our experiments in terms of the dataset, setup and the details of the baselines used. The performance of our approach is then compared to the state of the art baselines and our findings are discussed.

\subsubsection{Dataset and Experimental Setup}

Our experiments were conducted on a dataset collected from the popular location-based social network of Gowalla introduced in [26]. It includes check-in data (longitude, latitude, timestamp, categories, among others.) of more than 600,000 users from Austin, Chicago, Houston, Los Angeles and San Francisco. For each user, we randomly select $70 \%$ of her check-ins to construct the training data and the remaining $30 \%$ of her check-ins for testing data as suggested in [26]. 
We compare our approach with several state of the art point-of-interest recommendation methods that are briefly described in the following:

1. BasicMF is a classical matrix factorization techniques, which only considers users' past venue check-ins and, hence their preferences, for Point-of-Interest recommendation.

2. GeoCF $[48]$ is based on both user preference and geographical influence which are integrated into a collaborative filtering model.

3. MGMMF[6] is a framework based on Multi-Center Gaussian Model, which combines both the user preference and MGM based check-in probability for Point of interest recommendation.

4. Markov[7] applies Mobility Markov Chain model for predicting next venue of a user based on her mobility behavior over different time intervals and the recent venues that she has visited.

5. ML[30] considers user mobility, global mobility and temporal features to describe users' check-in behavior and applies M5 decision tree to predict the next POIs of a user.

6. CPOIR is one of the most related work in the literature by Liu et al [26], which proposes a Category-aware Point-Of-Interest Recommendation model that exploits the transition behavior of users between venue categories. They employ a matrix factorization model to predict the transition patterns of users' interests over categories and consequently her interests in different venues.

For evaluation purposes, we measure the performance of the methods based on Precision@K, Recall@K and F1-score as suggested in [26].

\subsubsection{Experimental Results}

In this section, in-line with [26], we compare the performance of our proposed approach with other state of the art baselines when Top- 6 venues are recommended by each method. The results are reported in Table 4.12 in terms of Recall, Precision and F1-score. 
It can be observed that BasicMF model, which is solely based on user interests performs worse than others for most of the cities and in terms of all three metrics. This means that incorporating other auxiliary information such as geographical, social, and temporal features leads to improved quality of venue recommendation. Markov models that incorporate temporal features outperform BasicMF; however, they perform much less accurately than the other baselines. This is because Markov models assume that a user's mobility data is dense, as a result they may not perform so well on users' venue data in LBSNs which is very sparse.

As another observation, MGMMF and GeoCF that fuse geographical influence and user interest into their proposed approach and take into account the correlation between these features offer more accurate recommendations compared to the ML model, which exploits geographical influence as a single feature. As mentioned before, the CPOIR model incorporates two novel features, i.e., the transition patterns of a user's interests among venues and venue categories, for the purpose of point-of-interest recommendation. It can be seen that CPOIR offers superior results compared to both MGMMF and GeoCF in all cities and in terms of all metrics. This demonstrates the benefits of these two factors to improve the quality of venue recommendations.

Inspired from these insights, in order to be able to utilize the benefits of useful features in a unified model for point-of-interest recommendation, we formalize user LBSN information in the form of a heterogeneous graph. As highlighted in Table 4.12, it is evident that our proposed approach outperforms all the comparison methods in all cities in terms of Precision and F1-score. This means that our approach can successfully take advantage of different features, i.e., venue categories, geographical influence, the relationship between users, and the correlation between these features to produce more accurate recommendations, i.e., less false positive. However, it can be observed that our proposed approach results in lower Recall values, i.e., more false negatives compared to others. In other words, our method is not able to identify those venues through the current limited set of defined meta-paths in this paper. To cover more meta-paths, as our future work, we intend to increase the length constraint of meta-paths, which 
is currently set to 3 , and take into account more features in our representation model such as transition of user's interests and sentiments of user-venue related comments.

\subsection{Neural Embedding Method}

\subsubsection{Dataset and Evaluation Methodology}

Our experiments were conducted on a dataset collected from the Gowalla LBSN introduced in [26]. Table 4.1 shows some statistics about the number of users and venues in each city. Since, each city has a different number of check-ins in our evaluation, the impact of the features is not always to the same degree. Our evaluation strategy and metrics are based on [26], which suggests to randomly select $70 \%$ of the check-ins of each user as the training data and leave the rest of the check-ins for testing. Further, the quality of the recommendations is measured based on Precision@6, Recall@6 and F1-Score. In terms of the parameters of our model, we train our embeddings based on an embedding dimension size of 100 . Further, we perform 10-fold cross-validation and select $N=5$, which is the best performing value for $N$ in top- $N$ similar user and venue features. The hyperparameters of the feature-based matrix factorization model were set as suggested in [9]. Namely, the learning rate, the regularization parameter for the user factor, the regularization parameter for the venue factor and the number of latent factors were set to $0.005,0.004,0.004$ and 64 .

\begin{tabular}{|c|c|c|c|c|}
\hline City & Users & Venues & checkins/user & checkins/venue \\
\hline Austin & 339 & 7936 & 138.226 & 5.905 \\
\hline Chicago & 257 & 1704 & 18.732 & 2.825 \\
\hline Houston & 163 & 6812 & 162.135 & 3.880 \\
\hline Los Angeles & 280 & 3607 & 43.179 & 3.352 \\
\hline San Francisco & 370 & 5447 & 62.405 & 4.239 \\
\hline
\end{tabular}

Table 4.1: Number of users and venues per city 


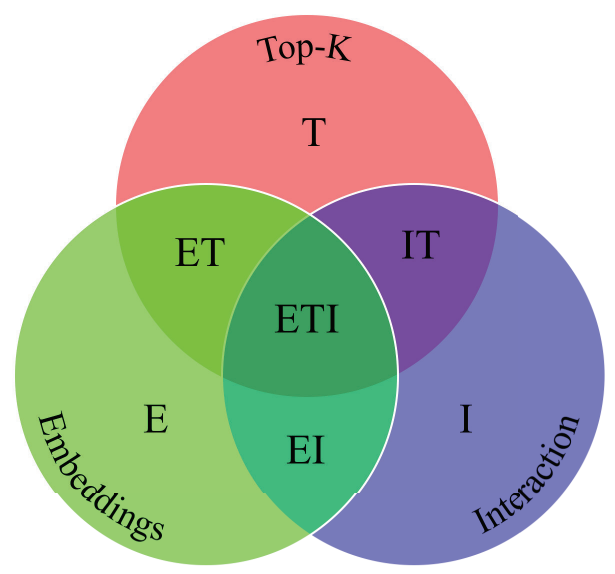

Figure 4.1: Seven variants based on our proposed features.

\subsubsection{Features Analysis}

In this section, we comprehensively evaluate the performance of the different features that we have proposed in this paper. We have proposed three types of features, i.e., user, venue and global user-venue interaction. Therefore, by selecting and combining the different alternatives for each feature, we obtain seven variants that we will systematically compare in this section. These seven variants are illustrated in Figure 4.1. For example, the method named ETI means that all the introduced features are encoded in the model and similarly EI means that only embedding vectors are included as well as the global user-venue interaction feature but the top- $\mathrm{N}$ features are not included.

The results of comparing different variants of our model are reported in terms of Precision@6 and Recall@6 in Figures 4.2 and 4.3 based on the 70-30 split that was mentioned earlier. As depicted in these figures, it can be observed that embedding features show the lowest performance. We believe the reason for this is that the number of embedding features is quite high (200 for user and venue features together) and each of the features does not convey any meaning in isolation. Therefore, the high number of highly dependent features leads to poor prediction. However, when merged with the top- $\mathrm{N}$ similar features the quality of the results improve. This observation shows that highly similar users and venues, regardless of their social connection to the user of interest, can serve as strong indicators of relevant 
venues for a user. It can also be seen in the results that when these two features are combined with the global feature, the results do not show any noticeable improvement.

\section{Precision}

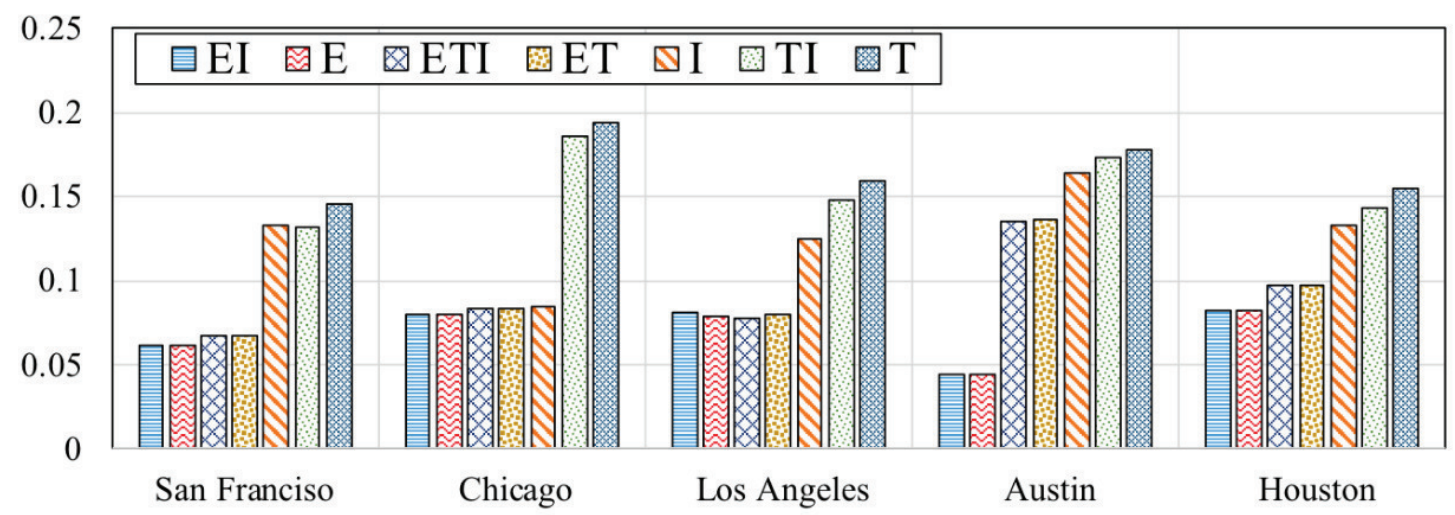

Figure 4.2: The Precision@6 of the seven variants of our proposed approach.

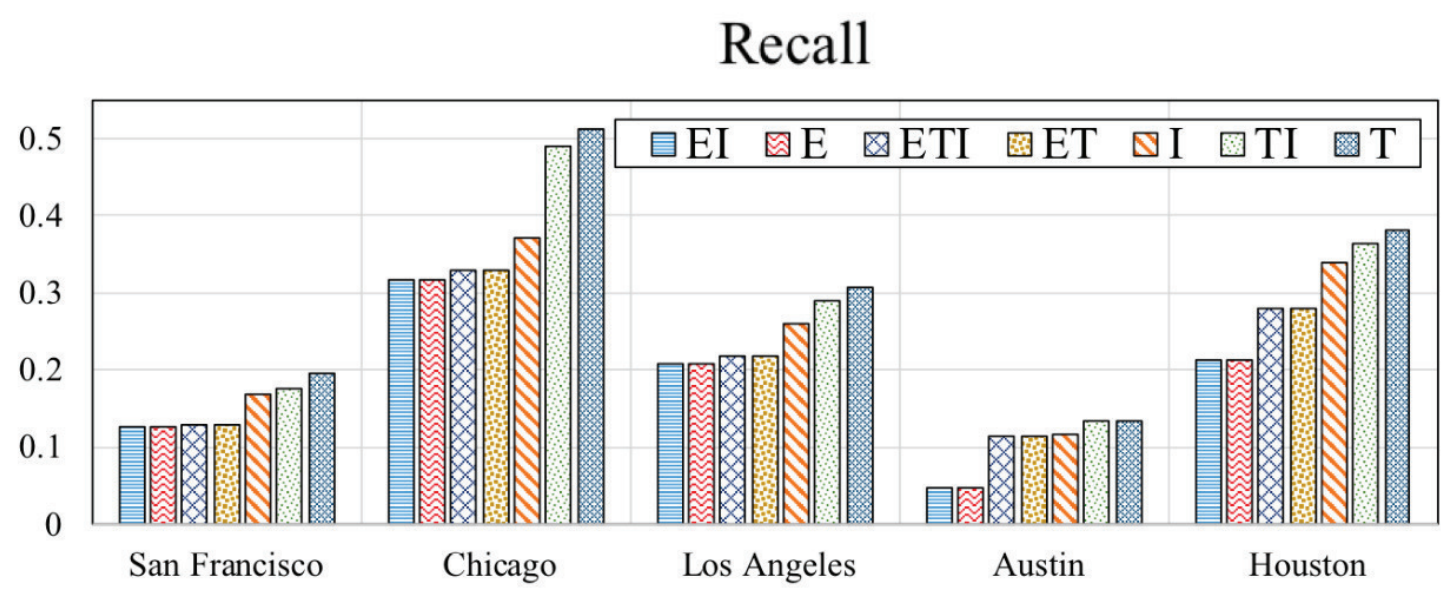

Figure 4.3: The Recall@6 of the seven variants of our proposed approach.

On the other hand, when global feature is used alone or in combination with the top- $\mathrm{N}$ similar features, the accuracy of venue recommendation increases. We conclude that the top- $\mathrm{N}$ similar features are the most effective for Point of Interest recommendation due to finding similar users and venues that are located close to each other in the embedding space. Given the way the embeddings are learnt, this finding basically means that considering information from (1) similar users that have shown similar check-in behavior and (2) venues that have had similar user check-ins have the highest predictive power 
for point-of-interest prediction. So in summary, we find:

1. Among the features, top-N similar users and venues serve as the best performing features compared to the other features.

2. The best variant that benefits from more than one feature is the "TI" model that uses both Top$\mathrm{N}$ similar features and the interaction between them, but it still has a weaker performance when compared to top- $\mathrm{N}$ similar features.

These findings show that our approach for embedding users and venues in the same space has been able to identify users with similar check-in patterns and venues with similar user check-ins behavior. Given the effective embedding of the users and venues, the consideration of top- $\mathrm{N}$ features and the global user-venue interaction feature based on these embeddings will point to the users that have similar behavioral patterns and so can be used for recommending the best point-of-interest. Based on this observation and from among the different variants analyzed in this section, we select model $\mathrm{T}$ as the point-of-interest recommendation model to be compared to the other baselines in the next section.

\subsubsection{Comparison with baselines}

For comparison, we use those baselines that have already used the same benchmark dataset and the same evaluation strategy for all of our models. In addition, we also compare this model's results with the Heterogeneous Link Prediction Model (covered in the last section) which formulates the problem as heterogeneous link prediction over venue categories, venue regions and user relationships in addition to historical check-in data of users. We perform our comparative analysis based on the strategy proposed in [26] and so only the top-6 venues are taken into consideration. We report precision, recall, and f-measure in Table 4.12.

This model outperforms all of the previous models which shows how significant these features are for venue recommendation. The Heterogeneous Link Prediction model, which uses link prediction for venue 
recommendation achieves better results compared to CPOIR model in terms of precision. However, it shows lower Recall compared to the others.

As reported in Table 4.12, our proposed model outperforms all the comparison methods in all three metrics. This observation indicates that by embedding user and venue vectors into the same space, a new set of effective features can be developed that are suitable for venue recommendation. By presenting the users and venues into the same space, not only closer users and locations are discovered, but also similar user-location pairs are found. We found that direct social connection between users is not necessarily an accurate indicator for venue recommendation. This is because users' social connections do not directly translate into visiting the same venues. In contrast, we developed an alternative feature for finding top- $N$ similar users and venues for each user and venue, which might not even be directly connected to the user or the venue. These top users and venues have similar temporal check-in behavior and as such have a similar embedding vector. The incorporation of these top-N users and venues substantially increases performance.

\subsection{Ensemble Method}

\subsubsection{Dataset and Evaluation Methodology}

Similarly to the previous evaluations, our experiments were conducted on a dataset collected from the Gowalla LBSN. Our evaluation strategy and metrics are based on [26], which indicates to randomly select $70 \%$ of the check-ins of each user as the training information and leave the remainder of the check-ins for testing. Additionally, the quality of the recommendations are assessed based on Precision@6, Recall@6 and F1-Score.

The top $-n$ similarity measure, as discussed before, has been divided into two categories, top-n embedded based similarity and meta-path based similarity. The embeddings have been trained based on an embedding dimension size of 100. In addition, similar to Section 4.2, we have selected the best 
performing value of $\mathrm{N}=5$ for users and venues by performing 10 -fold cross validation. For training feature-based matrix factorization model, the hyperparameters are set to similar values as Section 4.2.

\subsubsection{Ablation Study}

In this section, we comprehensively study the impact of the various features which we have proposed in this thesis. Results are summarized in Tables 4.3 to 4.12 . Similar to our previous model, we have proposed three types of features of user, venue and global user-venue interaction. However, we have evaluated the features by doing an ablation study, which refers to the removal of some "feature" of our model, and discovering precisely how that affects performance.

Since, there are three important factors, users, locations and user-location interaction which immediately impact users' personal preferences over locations, we have performed ablation study on our model and gathered Precision@6, Recall@6 and F1-Score@6 based on the 70/30 split that was mentioned before. Unlike our previous evaluations, we have trained and tested our model in three stages to compare the feature's influence on the results in an isolated approach.

For Users and Venues ablation study, by selecting and also mixing the various alternatives for every feature, we get 5 variants that we'll systematically compare in this section which are based on the features illustrated in Table 4.2.

\begin{tabular}{|c|c|}
\hline Variant & Meaning \\
\hline E & User or Venue Embedding \\
\hline TE & Top-N Embedded based Similarity \\
\hline TS & Top-N Meta-Path based Similarity \\
\hline I & Embedded based User and Venue Similarity \\
\hline PS & Meta-path based User and Venue Similarity \\
\hline
\end{tabular}

Table 4.2: Five variants based on our proposed features

First, for user and venue features, when the user and venue embeddings (E) is used solely for Pointof-Interest recommendation, we validated the hyptohesis that we have discussed in Section 4.2, that 
there is no significant improvement in the results. Next, we studied the results of the similarity features described earlier. As seen in the third row of Tables 4.3 to 4.8, we observed that by using Top-N MetaPath based Similarity (TS) instead of Top-N Embedding based Similarity (TE), the model outperforms most of the other features and achieves state-of-the-art results in terms of Precision@6, Recall@6 and F1-Score metrics.

Based on the gathered results from our last model and these features, it is clearly shown and the high number of dependent features does not improve the performance of Point-of-Interest recommendation. Therefore, as illustrated in the following tables, the embeddings features have the lowest performance effect on our model. However, top-N similarity features improve the results even when they are combined with the embedded features.

Hence, when the results of similarity measures are compared with each other, the quality of the results are improved considerably by using the meta-path based similarity between the nodes. Therefore, the top-N meta-path based similarity feature is the most effective for Point-of-Interest recommendation due to capturing the subtle semantics of similarity among peer objects in our Heterogeneous Information Network. It is seen that finding similar users and venues by using their distance in the same embedding space alone without using any additional data, cannot be the best solution. Also, for user and venue interaction, similar to other observations, meta-path based similarity between users and venues performs much better and it is a better solution than the embedding based similarity. 


\section{User Features}

\begin{tabular}{|l|l|l|l|l|l|l|l|}
\hline E & TE & TS & Austin & Chicago & Houston & LA & SF \\
\hline$\checkmark$ & & & 0.187 & 0.174 & 0.175 & 0.142 & 0.179 \\
\hline & $\checkmark$ & & 0.265 & 0.102 & 0.140 & 0.200 & 0.262 \\
\hline & & $\checkmark$ & 0.273 & 0.208 & 0.184 & 0.195 & 0.213 \\
\hline$\checkmark$ & $\checkmark$ & & 0.111 & 0.083 & 0.162 & 0.176 & 0.111 \\
\hline$\checkmark$ & & $\checkmark$ & 0.212 & 0.187 & 0.181 & 0.146 & 0.207 \\
\hline
\end{tabular}

Table 4.3: Precision@6 for User Features

\begin{tabular}{|c|c|c|c|c|c|c|c|}
\hline E & TE & TS & Austin & Chicago & Houston & LA & SF \\
\hline$\checkmark$ & & & 0.132 & 0.470 & 0.422 & 0.307 & 0.236 \\
\hline & $\checkmark$ & & 0.180 & 0.246 & 0.305 & 0.309 & 0.293 \\
\hline & & $\checkmark$ & 0.188 & 0.535 & 0.465 & 0.359 & 0.279 \\
\hline$\checkmark$ & $\checkmark$ & & 0.071 & 0.330 & 0.385 & 0.269 & 0.134 \\
\hline$\checkmark$ & & $\checkmark$ & 0.151 & 0.493 & 0.427 & 0.305 & 0.285 \\
\hline
\end{tabular}

Table 4.4: Recall@6 for User Features

\begin{tabular}{|c|c|c|c|c|c|c|c|}
\hline E & TE & TS & Austin & Chicago & Houston & LA & SF \\
\hline$\checkmark$ & & & 0.155 & 0.254 & 0.247 & 0.194 & 0.204 \\
\hline & $\checkmark$ & & 0.214 & 0.144 & 0.192 & 0.243 & 0.277 \\
\hline & & $\checkmark$ & 0.223 & 0.300 & 0.264 & 0.253 & 0.242 \\
\hline$\checkmark$ & $\checkmark$ & & 0.087 & 0.133 & 0.228 & 0.213 & 0.121 \\
\hline$\checkmark$ & & $\checkmark$ & 0.176 & 0.271 & 0.254 & 0.197 & 0.240 \\
\hline
\end{tabular}

Table 4.5: F1-Score for User Features 


\section{Venue Features}

\begin{tabular}{|l|l|l|l|l|l|l|l|}
\hline E & TE & TS & Austin & Chicago & Houston & LA & SF \\
\hline$\checkmark$ & & & 0.050 & 0.081 & 0.092 & 0.073 & 0.075 \\
\hline & $\checkmark$ & & 0.393 & 0.232 & 0.241 & 0.254 & 0.309 \\
\hline & & $\checkmark$ & 0.395 & 0.242 & 0.244 & 0.284 & 0.346 \\
\hline$\checkmark$ & $\checkmark$ & & 0.079 & 0.076 & 0.087 & 0.077 & 0.079 \\
\hline$\checkmark$ & & $\checkmark$ & 0.072 & 0.084 & 0.095 & 0.078 & 0.061 \\
\hline
\end{tabular}

Table 4.6: Precision@6 for Venue Features

\begin{tabular}{|c|c|c|c|c|c|c|c|}
\hline E & TE & TS & Austin & Chicago & Houston & LA & SF \\
\hline$\checkmark$ & & & 0.053 & 0.321 & 0.243 & 0.220 & 0.151 \\
\hline & $\checkmark$ & & 0.235 & 0.553 & 0.489 & 0.397 & 0.338 \\
\hline & & $\checkmark$ & 0.232 & 0.526 & 0.507 & 0.446 & 0.385 \\
\hline$\checkmark$ & $\checkmark$ & & 0.072 & 0.301 & 0.248 & 0.233 & 0.158 \\
\hline$\checkmark$ & & $\checkmark$ & 0.057 & 0.324 & 0.309 & 0.237 & 0.033 \\
\hline
\end{tabular}

Table 4.7: Recall@6 for Venue Features

\begin{tabular}{|l|l|l|l|l|l|l|l|}
\hline E & TE & TS & Austin & Chicago & Houston & LA & SF \\
\hline$\checkmark$ & & & 0.051 & 0.129 & 0.133 & 0.110 & 0.100 \\
\hline & $\checkmark$ & & 0.294 & 0.327 & 0.323 & 0.310 & 0.323 \\
\hline & & $\checkmark$ & 0.292 & 0.331 & 0.329 & 0.347 & 0.364 \\
\hline$\checkmark$ & $\checkmark$ & & 0.075 & 0.121 & 0.129 & 0.116 & 0.105 \\
\hline$\checkmark$ & & $\checkmark$ & 0.064 & 0.133 & 0.145 & 0.117 & 0.043 \\
\hline
\end{tabular}

Table 4.8: F1-Score for Venue Features 


\section{User-Venue Similarity Features}

\begin{tabular}{|c|c|c|c|c|c|c|}
\hline I & PS & Austin & Chicago & Houston & LA & SF \\
\hline$\checkmark$ & & 0.164 & 0.084 & 0.133 & 0.125 & 0.133 \\
\hline & $\checkmark$ & 0.320 & 0.265 & 0.273 & 0.312 & 0.292 \\
\hline
\end{tabular}

Table 4.9: Precision@6 for User-Venue Similarity Features

\begin{tabular}{|c|c|c|c|c|c|c|}
\hline I & PS & Austin & Chicago & Houston & LA & SF \\
\hline$\checkmark$ & & 0.116 & 0.272 & 0.340 & 0.259 & 0.169 \\
\hline & $\checkmark$ & 0.213 & 0.504 & 0.631 & 0.475 & 0.431 \\
\hline
\end{tabular}

Table 4.10: Recall@6 for User-Venue Similarity Features

\begin{tabular}{|c|c|c|c|c|c|c|}
\hline I & PS & Austin & Chicago & Houston & LA & SF \\
\hline$\checkmark$ & & 0.136 & 0.117 & 0.191 & 0.169 & 0.149 \\
\hline & $\checkmark$ & 0.255 & 0.347 & 0.381 & 0.377 & 0.348 \\
\hline
\end{tabular}

Table 4.11: F1-Score for User-Venue Similarity Features

\subsubsection{Comparison with Baselines}

For the purpose of comparison, we utilize a number of strong baselines that have been used in our previous evaluations and have also already used a similar benchmark dataset and used the identical evaluation approach to evaluate the work of ours with. We only take the top- 6 venues into consideration and report precision, recall and f-measure based on the top- 6 venues. As discussed before, we take the top-performing features and gather the final results of our model's point-of-interest recommendation. Based on the achieved results, the top performing features were Top-N Meta-Path Based Similarity (TS) for Users and Venues and Meta-path based User and Venue Similarity (PS). The comparison of our results with other state-of-the-art approaches are reported in the following table. 


\begin{tabular}{|c|c|c|c|c|c|c|c|c|c|c|c|c|c|c|c|}
\hline \multirow{2}{*}{ City } & \multicolumn{3}{|c|}{ Austin } & \multicolumn{3}{|c|}{ Chicago } & \multicolumn{3}{|c|}{ Houston } & \multicolumn{3}{|c|}{ Los Angeles } & \multicolumn{3}{|c|}{ San Francisco } \\
\hline & $\mathrm{R}$ & $\mathrm{P}$ & $\mathrm{F} 1$ & $\mathrm{R}$ & $\mathrm{P}$ & $\mathrm{F} 1$ & $\mathrm{R}$ & $\mathrm{P}$ & F1 & $\mathrm{R}$ & $\mathrm{P}$ & F1 & $\mathrm{R}$ & $\mathrm{P}$ & F1 \\
\hline Ensemble Features Method & 0.214 & 0.200 & 0.207 & 0.671 & 0.248 & 0.362 & 0.774 & 0.349 & 0.481 & 0.634 & 0.280 & 0.388 & 0.438 & 0.280 & 0.342 \\
\hline Neural Embedding & 0.134 & 0.178 & 0.153 & 0.513 & 0.194 & 0.282 & 0.380 & 0.155 & 0.220 & 0.306 & 0.159 & 0.153 & 0.195 & 0.146 & 0.167 \\
\hline Heterogeneous Link Prediction & 0.056 & 0.096 & 0.071 & 0.096 & 0.130 & 0.111 & 0.207 & 0.122 & 0.153 & 0.120 & 0.112 & 0.116 & 0.072 & 0.116 & 0.089 \\
\hline CPOIR [26] & 0.157 & 0.026 & 0.045 & 0.292 & 0.049 & 0.083 & 0.279 & 0.046 & 0.080 & 0.203 & 0.034 & 0.058 & 0.159 & 0.027 & 0.045 \\
\hline BasicMF & 0.064 & 0.011 & 0.018 & 0.086 & 0.014 & 0.025 & 0.082 & 0.014 & 0.024 & 0.072 & 0.012 & 0.021 & 0.066 & 0.011 & 0.019 \\
\hline GeoCF[48] & 0.122 & 0.020 & 0.035 & 0.227 & 0.038 & 0.065 & 0.165 & 0.027 & 0.047 & 0.164 & 0.027 & 0.047 & 0.126 & 0.021 & 0.036 \\
\hline MGMMF[6] & 0.117 & 0.020 & 0.034 & 0.186 & 0.031 & 0.053 & 0.159 & 0.027 & 0.045 & 0.152 & 0.025 & 0.046 & 0.112 & 0.019 & 0.032 \\
\hline Markov[7] & 0.086 & 0.014 & 0.025 & 0.116 & 0.019 & 0.033 & 0.102 & 0.017 & 0.029 & 0.096 & 0.016 & 0.027 & 0.088 & 0.015 & 0.025 \\
\hline $\operatorname{ML}[30]$ & 0.116 & 0.019 & 0.033 & 0.170 & 0.028 & 0.049 & 0.152 & 0.025 & 0.044 & 0.132 & 0.022 & 0.038 & 0.111 & 0.018 & 0.032 \\
\hline
\end{tabular}

Table 4.12: All models comparative analysis with the baselines divided across different cities.

As mentioned before, BasicMF produces the lowest results because it is solely based on user interests. Therefore, when rich information such as social, geographical and temporal features are encoded to the model, quality of Point-of-Interest recommendations is improved. This is clearly seen and validated from Markov model's results which incorporates temporal features. However, both of these models have achieved the lowest recommendation results compared to other state-of-art models since they assume user's mobility data is dense. Our observation of encoding rich information into the Point-of-Interest recommendation is also proven by looking at other models such as MGMMF and GeoCF results which are more accurate recommendations compared to the ML model.

As highlighted in Table 4.12, it is evident that our final proposed approach outperforms all the comparison models in all cities in terms of Precision, Recall and F1-Score. This means that our approach can successfully take the advantage of different features (relationship between users, category of locations,etc.) and produce more accurate recommendations.

In addition, compared to our second proposed approach, our ensemble method is outperforming the top-k embedding based similarity results due to taking more features into account. This is done by using the metapaths defined in Tables 3.2 and 3.3 and finding the similarity between users and venues based on various properties. Thus, finding the similarity between users and venues based on their 
generated embeddings from their IDs in the same space is not the best solution for point-of-interest recommendations. Since the metapath-based similarity framework finds the similarity between the users and venues by going through the defined meta-paths, more auxiliary information is used for making recommendations to the users. For instance, it can be observed that embedding users and venues into the same space by using their IDs does not take the categorical and geographical information about the user and venue into account. As seen in Tables 4.3 to 4.8, the recommendations created from the pair of Embeddings (E) and Top-n metapath-based similar users and venues (TS) have generated higher results than the pair of Embeddings (E) and Top-n embedding-based similar users and venues (TS). 


\section{Chapter 5}

\section{Conclusion}

The focus of this thesis has been to provide point-of-interest recommendation by viewing it as an instance of the link prediction problem on heterogeneous graphs and learning neural embeddings from users historical check-in sequences. As such, we have systematically shown how various types of information can be incorporated into a heterogeneous graph based on which distance metrics between nodes can be employed as features to learn ranking classifiers. We find that even a logistic regression method can effectively show competitive performance compared to the state of the art despite its simplicity and light-weight computational requirements.

We also show how sequence of user check-ins can be used to learn user and venue representations within the same embedding space that are then used to introduce three types of features. Our analysis of earlier methods had shown that the real social network of each user does not necessarily indicate similar venue recommendations and as such we build user relationships based on finding the top- $\mathrm{N}$ meta-path based similar users and venues to the given user within the embedding space. This feature showed to have the strongest performance for predicting a point-of-interest. Finally, we also considered the raw embedding representation of users and venues to serve as features, which did not show a good performance. 
We also systematically explored the possibility of building models that use a combination of these methods and found that the top- $\mathrm{N}$ meta-path based similar features are the strongest features both in isolation and when combined with other features. We also showed that compared to the state of the art methods, which benefit from a wide range of social, geographical and content features, that our variant that only relies on top- $\mathrm{N}$ meta-path based similarity features shows a noticeably better performance. We further found that our proposed approach shows improved performance over the baselines in terms of all metrics of Precision, Recall and F1-Score.

\section{$5.1 \quad$ Future Work}

Based on the evaluations that we have reported in this thesis and the comparison with the current stateof-the-art approaches, it is clearly seen that our proposed approaches are the effective Point-of-Interest recommendation models. Our future work will explore various synergistic directions:

We will explore whether a more extensive set of meta-paths defined over the network schema can lead to improved recall for the Heterogeneous Link Prediction method or not. Currently, we have narrowed our meta-paths length to four. By increasing the amount of metapaths our meta-path based similarity framework would take more paths into consideration and consider similarity between users and venues from different aspects.

As mentioned before, recently there have been various point-of-interest recommendation models. However, we are interested in the possibility of making recommendation by using heterogeneous link prediction and Sun's approach [39] to recommend venues at a certain time. Right now, we do not take time into consideration for making recommendations and this can be an interesting topic to research on for future.

In addition, since users may visit new locations in future, historical derived features such as the embeddings and top-k similarity fail to increase the accuracy of Point-of-Interest recommendations for 
these cases. New locations can be recommended to the users by mining users personalities [2] from their social network profiles. Similar to the cold-start users where there is no information to be used about their location preferences. We are also interested in the possibility of using users social-network profiles and the locations popularity among the cities for POI recommendations.

We are also interested in integrating our Point-of-Interest recommendation model data with advertisements [5] based on the locations that the user might visit in future. This could additionally help third-parties including advertisers to offer precise advertisements for the recommended positions.

Some users provide written textual feedback about their experience at venues they visit. These include textual reviews or recommendations. We are interested in the possibility of incorporating such unstructured user feedback into the network schema to see whether textual feedback, while quite sparse, can improve the venue recommendation task. 


\section{References}

[1] Gediminas Adomavicius and YoungOk Kwon. Improving aggregate recommendation diversity using ranking-based techniques. IEEE Trans. Knowl. Data Eng., 24(5):896-911, 2012.

[2] Aris Anagnostopoulos, Ravi Kumar, and Mohammad Mahdian. Influence and correlation in social networks. In Proceedings of the 14th ACM SIGKDD International Conference on Knowledge Discovery and Data Mining, Las Vegas, Nevada, USA, August 24-27, 2008, pages 7-15, 2008.

[3] Bokai Cao, Xiangnan Kong, and Philip S. Yu. Collective prediction of multiple types of links in heterogeneous information networks. In 2014 IEEE International Conference on Data Mining, ICDM 2014, Shenzhen, China, December 14-17, 2014, pages 50-59, 2014.

[4] Tianqi Chen, Weinan Zhang, Qiuxia Lu, Kailong Chen, Zhao Zheng, and Yong Yu. Svdfeature: a toolkit for feature-based collaborative filtering. Journal of Machine Learning Research, 13:3619$3622,2012$.

[5] Ye Chen, Michael Kapralov, Dmitry Pavlov, and John F. Canny. Factor modeling for advertisement targeting. In Advances in Neural Information Processing Systems 22: 23rd Annual Conference on Neural Information Processing Systems 2009. Proceedings of a meeting held 7-10 December 2009, Vancouver, British Columbia, Canada., pages 324-332, 2009. 
[6] Chen Cheng, Haiqin Yang, Irwin King, and Michael R. Lyu. Fused matrix factorization with geographical and social influence in location-based social networks. In Proceedings of the TwentySixth AAAI Conference on Artificial Intelligence, July 22-26, 2012, Toronto, Ontario, Canada., 2012.

[7] S. Gambs, M.O. Killijian, and M.N. Prado Cortez. Next place prediction using mobility markov chains. In Eurosys' MPM, 2012.

[8] Sébastien Gambs, Marc-Olivier Killijian, and Miguel Núñez del Prado Cortez. Next place prediction using mobility markov chains. In Proceedings of the First Workshop on Measurement, Privacy, and Mobility - MPM 12. ACM Press, 2012.

[9] Tao Ge, Zhifang Sui, and Baobao Chang. Exploiting collaborative filtering techniques for automatic assessment of student free-text responses. In CIKM, pages 1493-1496, 2013.

[10] Genevieve Gorrell and Brandyn Webb. Generalized hebbian algorithm for incremental latent semantic analysis. In INTERSPEECH 2005 - Eurospeech, 9th European Conference on Speech Communication and Technology, Lisbon, Portugal, September 4-8, 2005, pages 1325-1328, 2005.

[11] Qing Guo, Zhu Sun, Jie Zhang, Qi Chen, and Yin-Leng Theng. Aspect-aware point-of-interest recommendation with geo-social influence. In Adjunct Publication of the 25th Conference on User Modeling, Adaptation and Personalization, UMAP 2017, Bratislava, Slovakia, July 09 - 12, 2017, pages $17-22,2017$.

[12] Siyao Han and Yan Xu. Link prediction in microblog network using supervised learning with multiple features. JCP, 11(1):72-82, 2016.

[13] Daniel S. Himmelstein and Sergio E. Baranzini. Heterogeneous network edge prediction: A data integration approach to prioritize disease-associated genes. PLoS Computational Biology, 11(7), 2015. 
[14] Glen Jeh and Jennifer Widom. Simrank: a measure of structural-context similarity. In Proceedings of the Eighth ACM SIGKDD International Conference on Knowledge Discovery and Data Mining, July 23-26, 2002, Edmonton, Alberta, Canada, pages 538-543, 2002.

[15] Glen Jeh and Jennifer Widom. Scaling personalized web search. In Proceedings of the Twelfth International World Wide Web Conference, WWW 2003, Budapest, Hungary, May 20-24, 2003, pages 271-279, 2003.

[16] Leo Katz. A new status index derived from sociometric analysis. Psychometrika, 18(1):39-43, mar 1953.

[17] Yehuda Koren and Robert M. Bell. Advances in collaborative filtering. In Recommender Systems Handbook, pages 77-118. 2015.

[18] Yehuda Koren, Robert M. Bell, and Chris Volinsky. Matrix factorization techniques for recommender systems. IEEE Computer, 42(8):30-37, 2009.

[19] Quoc V. Le and Tomas Mikolov. Distributed representations of sentences and documents. In Proceedings of the 31th International Conference on Machine Learning, ICML 2014, Beijing, China, 21-26 June 2014, pages 1188-1196, 2014.

[20] Vincent Leroy, Berkant Barla Cambazoglu, and Francesco Bonchi. Cold start link prediction. In Proceedings of the 16th ACM SIGKDD International Conference on Knowledge Discovery and Data Mining, Washington, DC, USA, July 25-28, 2010, pages 393-402, 2010.

[21] Ying Li, Bin Cao, Lida Xu, Jianwei Yin, ShuiGuang Deng, Yuyu Yin, and Zhaohui Wu. An efficient recommendation method for improving business process modeling. IEEE Trans. Industrial Informatics, 10(1):502-513, 2014. 
[22] David Liben-Nowell and Jon M. Kleinberg. The link prediction problem for social networks. In Proceedings of the 2003 ACM CIKM International Conference on Information and Knowledge Management, New Orleans, Louisiana, USA, November 2-8, 2003, pages 556-559, 2003.

[23] David Liben-Nowell and Jon M. Kleinberg. The link-prediction problem for social networks. JASIST, 58(7):1019-1031, 2007.

[24] Ryan Lichtenwalter, Jake T. Lussier, and Nitesh V. Chawla. New perspectives and methods in link prediction. In Proceedings of the 16th ACM SIGKDD International Conference on Knowledge Discovery and Data Mining, Washington, DC, USA, July 25-28, 2010, pages 243-252, 2010.

[25] Xiaozhong Liu, Yingying Yu, Chun Guo, and Yizhou Sun. Meta-path-based ranking with pseudo relevance feedback on heterogeneous graph for citation recommendation. In Proceedings of the 23rd ACM International Conference on Conference on Information and Knowledge Management, CIKM 2014, Shanghai, China, November 3-7, 2014, pages 121-130, 2014.

[26] Xin Liu, Yong Liu, Karl Aberer, and Chunyan Miao. Personalized point-of-interest recommendation by mining users' preference transition. In 22nd ACM International Conference on Information and Knowledge Management, CIKM'13, San Francisco, CA, USA, October 27 - November 1, 2013, pages $733-738,2013$.

[27] Xin Luo, Mengchu Zhou, Yunni Xia, and Qingsheng Zhu. An efficient non-negative matrixfactorization-based approach to collaborative filtering for recommender systems. IEEE Trans. Industrial Informatics, 10(2):1273-1284, 2014.

[28] Tomas Mikolov, Kai Chen, Greg Corrado, and Jeffrey Dean. Efficient estimation of word representations in vector space. CoRR, abs/1301.3781, 2013.

[29] M. E. J. Newman. Clustering and preferential attachment in growing networks. Physical Review E, 64(2), jul 2001 
[30] Anastasios Noulas, Salvatore Scellato, Neal Lathia, and Cecilia Mascolo. Mining user mobility features for next place prediction in location-based services. In 12th IEEE International Conference on Data Mining, ICDM 2012, Brussels, Belgium, December 10-13, 2012, pages 1038-1043, 2012.

[31] Dan Pelleg and Andrew W. Moore. X-means: Extending k-means with efficient estimation of the number of clusters. In Proceedings of the Seventeenth International Conference on Machine Learning (ICML 2000), Stanford University, Stanford, CA, USA, June 29 - July 2, 2000, pages $727-734,2000$.

[32] Tuan-Anh Nguyen Pham, Xutao Li, and Gao Cong. A general model for out-of-town region recommendation. In Proceedings of the 26th International Conference on World Wide Web, WWW 2017, Perth, Australia, April 3-7, 2017, pages 401-410, 2017.

[33] Alireza Pourali, Fattane Zarrinkalam, and Ebrahim Bagheri. Point-of-interest recommendation using heterogeneous link prediction. In Proceedings of the 21th International Conference on Extending Database Technology, EDBT 2018, Vienna, Austria, March 26-29, 2018., pages 481-484, 2018.

[34] Xingyi Ren, Meina Song, Haihong E, and Junde Song. Context-aware probabilistic matrix factorization modeling for point-of-interest recommendation. Neurocomputing, 241:38-55, jun 2017.

[35] Chuan Shi, Yitong Li, Jiawei Zhang, Yizhou Sun, and Philip S. Yu. A survey of heterogeneous information network analysis. IEEE Trans. Knowl. Data Eng., 29(1):17-37, 2017.

[36] Yizhou Sun, Charu C. Aggarwal, and Jiawei Han. Relation strength-aware clustering of heterogeneous information networks with incomplete attributes. PVLDB, 5(5):394-405, 2012.

[37] Yizhou Sun, Rick Barber, Manish Gupta, Charu C. Aggarwal, and Jiawei Han. Co-author relationship prediction in heterogeneous bibliographic networks. In International Conference on Advances in Social Networks Analysis and Mining, ASONAM 2011, Kaohsiung, Taiwan, 25-27 July 2011, pages 121-128, 2011. 
[38] Yizhou Sun and Jiawei Han. Mining heterogeneous information networks: a structural analysis approach. SIGKDD Explorations, 14(2):20-28, 2012.

[39] Yizhou Sun, Jiawei Han, Charu C. Aggarwal, and Nitesh V. Chawla. When will it happen?: relationship prediction in heterogeneous information networks. In Proceedings of the Fifth International Conference on Web Search and Web Data Mining, WSDM 2012, Seattle, WA, USA, February 8-12, 2012, pages 663-672, 2012.

[40] Yizhou Sun, Jiawei Han, Xifeng Yan, Philip S. Yu, and Tianyi Wu. Pathsim: Meta path-based top-k similarity search in heterogeneous information networks. PVLDB, 4(11):992-1003, 2011.

[41] Chao Wang, Venu Satuluri, and Srinivasan Parthasarathy. Local probabilistic models for link prediction. In Proceedings of the 7th IEEE International Conference on Data Mining (ICDM 2007), October 28-31, 2007, Omaha, Nebraska, USA, pages 322-331, 2007.

[42] Jian Wei, Jianhua He, Kai Chen, Yi Zhou, and Zuoyin Tang. Collaborative filtering and deep learning based hybrid recommendation for cold start problem. In 2016 IEEE 14th Intl Conf on Dependable, Autonomic and Secure Computing, 14th Intl Conf on Pervasive Intelligence and Computing, 2nd Intl Conf on Big Data Intelligence and Computing and Cyber Science and Technology Congress, DASC/PiCom/DataCom/CyberSciTech 2016, Auckland, New Zealand, August 8-12, 2016, pages 874-877, 2016.

[43] Ming-Fang Weng and Yung-Yu Chuang. Collaborative video reindexing via matrix factorization. TOMCCAP, 8(2):23:1-23:20, 2012.

[44] Zhipeng Wu, Hui Tian, Xuzhen Zhu, and Shuo Wang. Optimization matrix factorization recommendation algorithm based on rating centrality. In Data Mining and Big Data - Third International Conference, DMBD 2018, Shanghai, China, June 17-22, 2018, Proceedings, pages 114-125, 2018. 
[45] Min Xie, Hongzhi Yin, Hao Wang, Fanjiang Xu, Weitong Chen, and Sen Wang. Learning graphbased POI embedding for location-based recommendation. In Proceedings of the 25th ACM International Conference on Information and Knowledge Management, CIKM 2016, Indianapolis, IN, USA, October 24-28, 2016, pages 15-24, 2016.

[46] Dingqi Yang, Daqing Zhang, Zhiyong Yu, Zhiwen Yu, and Djamal Zeghlache. SESAME: mining user digital footprints for fine-grained preference-aware social media search. ACM Trans. Internet Techn., 14(4):28:1-28:24, 2014.

[47] Dingqi Yang, Daqing Zhang, Vincent W. Zheng, and Zhiyong Yu. Modeling user activity preference by leveraging user spatial temporal characteristics in lbsns. IEEE Trans. Systems, Man, and Cybernetics: Systems, 45(1):129-142, 2015.

[48] Mao Ye, Peifeng Yin, Wang-Chien Lee, and Dik Lun Lee. Exploiting geographical influence for collaborative point-of-interest recommendation. In Proceeding of the 34 th International ACM SIGIR Conference on Research and Development in Information Retrieval, SIGIR 2011, Beijing, China, July 25-29, 2011, pages 325-334, 2011.

[49] Yang Ye, Yu Zheng, Yukun Chen, Jianhua Feng, and Xing Xie. Mining individual life pattern based on location history. In MDM 2009, Tenth International Conference on Mobile Data Management, Taipei, Taiwan, 18-20 May 2009, pages 1-10, 2009.

[50] Quan Yuan, Gao Cong, Zongyang Ma, Aixin Sun, and Nadia Magnenat-Thalmann. Time-aware point-of-interest recommendation. In The 36th International ACM SIGIR conference on research and development in Information Retrieval, SIGIR '13, Dublin, Ireland - July 28 - August 01, 2013, pages $363-372,2013$. 
[51] Yin Zhang, Min Chen, Dijiang Huang, Di Wu, and Yong Li. idoctor: Personalized and professionalized medical recommendations based on hybrid matrix factorization. Future Generation Comp. Syst., 66:30-35, 2017.

[52] Wayne Xin Zhao, Sui Li, Yulan He, Edward Y. Chang, Ji-Rong Wen, and Xiaoming Li. Connecting social media to e-commerce: Cold-start product recommendation using microblogging information. IEEE Trans. Knowl. Data Eng., 28(5):1147-1159, 2016.

[53] Ning Zhou, William K. Cheung, Guoping Qiu, and Xiangyang Xue. A hybrid probabilistic model for unified collaborative and content-based image tagging. IEEE Trans. Pattern Anal. Mach. Intell., 33(7):1281-1294, 2011. 